\title{
A study of the low-frequency quasi-periodic oscillations in the X-ray light curves of the black hole candidate XTE J1859+226
}

\author{
P. Casella ${ }^{1,2, \star}$, T. Belloni ${ }^{3}$, J. Homan $^{4}$, and L. Stella ${ }^{1}$ \\ 1 INAF - Osservatorio Astronomico di Roma, Via di Frascati 33, 00040 Monte Porzio Catone, Roma, Italy \\ e-mail: casella@mporzio.astro.it \\ 2 Physics Department, Università degli Studi "Roma Tre", Via della Vasca Navale 84, 00146 Roma, Italy \\ 3 INAF - Osservatorio Astronomico di Brera, via E. Bianchi 46, 23807 Merate (LC), Italy \\ ${ }^{4}$ Center for Space Researh, Massachusetts Institute of Technology, 77 Massachusetts Avenue, Cambridge, MA 02139, USA
}

Received 5 May 2004 / Accepted 6 July 2004

\begin{abstract}
We present the results of an extensive timing analysis of the 1999 outburst of the soft X-ray transient and black hole candidate XTE J1859+226 as observed with the Rossi X-Ray Timing Explorer. Three main different types of low frequency (1-9 Hz) quasi-periodic oscillations (QPOs) were observed and classified, strengthening the general picture that is emerging for the variability properties of black hole X-ray binaires. Rapid transitions between different power spectral shapes were observed and their link with the count rate was studied. Furthermore, we show that a frequency of $\sim 6 \mathrm{~Hz}$ seems to hold a particular place: one of the three QPO types we found was very stable when at this frequency, as it happens in $\mathrm{Z}$ sources as well. The coherence of its subharmonic peak was higher when the fundamental was close to $6 \mathrm{~Hz}$, thus suggesting the presence of some resonance at this frequency.
\end{abstract}

Key words. X-rays: binaries - stars: individual: XTE J1859+226

\section{Introduction}

Observations with the Rossi X-ray Timing Explorer (RXTE) have led to an extraordinary progress in the knowledge of the variability properties of black-hole candidates (BHCs) in X-ray binaries (see e.g. van der Klis 2000; Remillard et al. 2002a). The fast quasi-periodic oscillations (QPOs) that were discovered in many of these systems are thought to originate in the innermost regions of the accretion flows around stellarmass black holes. Even though the mechanism responsible for the QPOs is still unknown, the study of their properties and behaviour can provide important clues on the physics of accretion onto BHCs.

While only a few BHCs show high-frequency QPOs $(50-450 \mathrm{~Hz}$, for a recent review see McClintock \& Remillard 2004, and references therein), low-frequency QPOs with frequencies ranging from a few $\mathrm{mHz}$ to $\sim 10 \mathrm{~Hz}$ are a common feature. The low-frequency QPOs were already known before the RXTE era (see van der Klis 1995 for an overview). While only a few detections were reported on the basis of EXOSAT data in the early 80 's, the Ginga satellite showed clear QPO features in the power density spectra (PDS) of BHCs (see van der Klis 1995). With RXTE, low-frequency QPOs were detected in

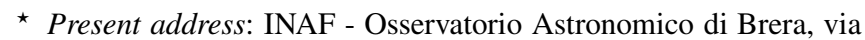
E. Bianchi 46, 23807 Merate (LC), Italy. virtually all observed BHCs (see van der Klis 2004). Usually these QPOs are associated with the spectrally hard and intermediate states (van der Klis 1995; McClintock \& Remillard 2004): they appear together with a flat-top noise component, and on a time scale of days their frequency often correlates with the source count rate (see e.g. Cui 1998; Reig et al. 2000). However, in the Ginga observations of the bright transient GS 1124-683 two distinct types of low frequency QPOs were identified: one associated with a flat-top noise component and one to a steep noise component (Takizawa et al. 1997). The first type showed a strong dependence on count rate, while the other had a rather stable frequency. These two QPOs had centroid frequencies in the 1-10 Hz range and were clearly related to the two PDS "flavors" of very high state observed in this system (Miyamoto et al. 1993).

Wijnands et al. (1999) and Homan et al. (2001) reported on two different types of QPOs in the RXTE data of XTE J1550-564: a broad one (type-A), with a quality factor $Q$ (the QPO frequency divided by the QPO full-widthhalf-maximum $(F W H M)$ ) of less than 3 , and a narrower one (type-B), with a $Q$ larger than 6. Both QPOs were characterized by a centroid frequency of $6 \mathrm{~Hz}$ and associated with a weak red-noise component, but with different phase-lag behaviours. XTE J1550-564 also showed the more common QPO-type associated with a flat-top noise component (see Cui et al. 1999; 


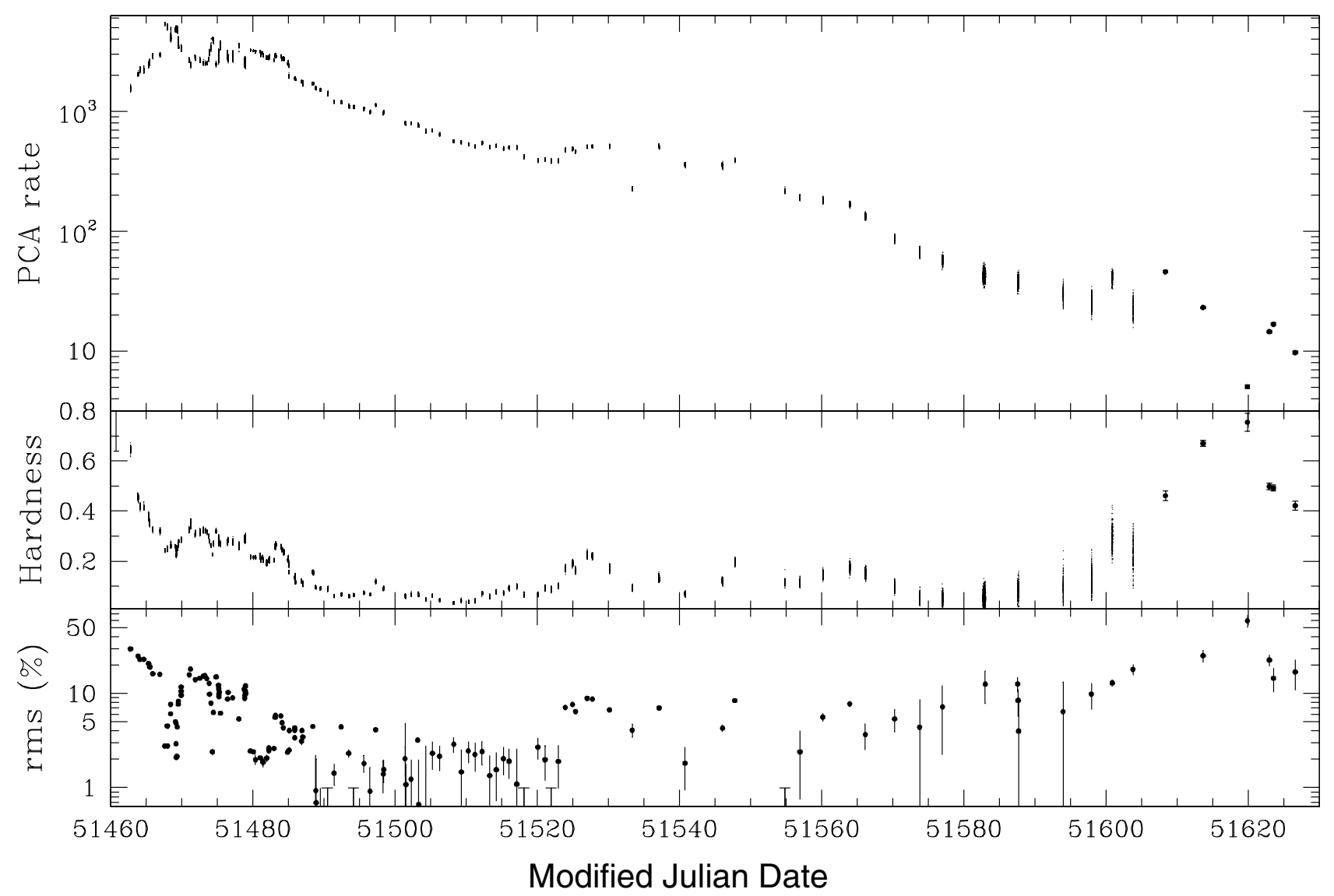

Fig. 1. RXTE/PCA light curve (PCUs 0 and 2, top panel), hardness ratio $((7-15 \mathrm{keV}) /(2-7 \mathrm{keV})$, middle panel) and total rms $(0.03-64 \mathrm{~Hz}$, bottom panel) of XTE J1859+226 during its 1999 outburst. The time resolution for the light- and hardness curves is $16 \mathrm{~s}$ except for the last five points, which have one point for each observation.

Sobczak et al. 2000). Remillard et al. (2002b) dubbed this QPO "type-C": its features are a high coherence $(Q \gtrsim 10)$, a variable centroid frequency (in the range $0.1-10 \mathrm{~Hz}$ ) and a strong flattop noise component ( $\sim 10-40 \%$ rms) (see Table 1 ).

While type-C QPOs are observed in many systems, the other two are less common. In addition to XTE J1550-564, type-B QPOs were also observed in GX 339-4 (e.g. Miyamoto et al. 1991; Nespoli et al. 2003), GRS 1739-278 (Wijnands et al. 2001) and possibly in 4U 1630-47 (Tomsick \& Kaaret 2000), while type-A QPOs were observed in GX 339-4 (Nespoli et al. 2003) and possibly in 4U 1630-47 (Tomsick \& Kaaret 2000; Dieters et al. 2000). Furthermore, in the light of the A-B-C classification, the two QPOs observed in GS 1124-683 (see above) can be tentatively identified with types $\mathrm{B}$ and $\mathrm{C}$, although a detailed analysis of Ginga data is necessary to confirm this association.

These oscillations, whose nature is still not understood, provide a direct way to explore the accretion flow around black holes (and neutron stars). In particular, their association with specific spectral states and the phenomenology that is emerging indicate that they are a key ingredient in understanding the physical conditions that give origin to the different states.

The soft X-ray transient XTE J1859+226 was discovered on 1999 October 9 with the RXTE All Sky Monitor (Wood et al. 1999), which detected a 2-12 X-ray flux of $\sim 160 \mathrm{mCrab}\left(\sim 4.5 \times 10^{-9} \mathrm{erg} \mathrm{s}^{-1} \mathrm{~cm}^{-2}\right)$ quickly rising at a
Table 1. Summary of type-A, -B and -C QPOs properties in XTE J1550-564 (Wijnands et al. 1999; Homan et al. 2001; Remillard et al. 2002b).

\begin{tabular}{lccc}
\hline \hline Property & Type A & Type B & Type C \\
\hline Frequency $(\mathrm{Hz})$ & $\sim 6$ & $\sim 6$ & $0.1-10$ \\
$Q(v / F W H M)$ & $\lesssim 3$ & $\gtrsim 6$ & $\gtrsim 10$ \\
Amplitude $(\% \mathrm{rms})$ & $3-4$ & $\sim 4$ & $3-16$ \\
Noise & weak red & weak red & strong flat-top \\
\hline
\end{tabular}

rate of $\sim 6 \mathrm{mCrab} / \mathrm{hour}$. A follow-up observation of the source with the RXTE/PCA (Proportional Counter Array, 2-60 keV) revealed a hard power-law dominated spectrum (Markwardt et al. 1999). On October 16, the source reached its peak flux of $\sim 5 \times 10^{-8} \mathrm{erg} \mathrm{s}^{-1} \mathrm{~cm}^{-2}$ in the $2-80 \mathrm{keV}$ band (corresponding to a luminosity of $\sim 7 \times 10^{38} \mathrm{erg} \mathrm{s}^{-1}$ for an assumed distance of $11 \mathrm{kpc}$ (Zurita et al. 2002)). After the initial hard rise, XTE J1859+226 softened at its peak intensity and continued to soften for almost two months, when a secondary hard plateau took place (see Fig. 1, MJD 51 520-51 540). This was rather similar to the behaviour observed in many X-ray transients, in particular XTE J1550-564 (see Remillard et al. 2002b, and references therein), suggesting a common scenario for the evolution of these objects. Both low-frequency $(\sim 1-4 \mathrm{~Hz}$ and $\sim 6 \mathrm{~Hz})$ 
and high-frequency $(\sim 150-187 \mathrm{~Hz})$ QPOs have been reported (see Cui et al. 2000; Focke et al. 2000) from XTE J1859+226.

The optical (Garnavich et al. 1999) and radio (Pooley \& Hjellming 1999) counterparts were identified soon after the discovery of the source. Optical photometry revealed a possible period of $9.15 \mathrm{~h}$ (Garnavich \& Quinn 2000). Monitoring at radio wavelengths suggested that at least two relativistic ejection episodes took place approximately on October 16.5 (MJD = $51467.5)$ and 27 (MJD = 51478). However, no ejecta were spatially resolved at radio wavelengths (Brocksopp et al. 2002).

Here we present the results of an extensive X-ray timing analysis of the 1999 outburst of XTE J1859+226, focussing on the low-frequency QPOs. We found three different types of low frequency $(1-10 \mathrm{~Hz}) \mathrm{QPOs}$. We show that these correspond to the above-mentioned A, B and C QPOs types, all of them showing distinctive and well defined behaviours and phase lags.

\section{Observations and data analysis}

We analyzed 129 RXTE/PCA observations made during the 1999 outburst of the black-hole candidate XTE J1859+226, between MJD 51462 (1999-10-11) and 51626 (2000-03-23). Table 2 shows dates and parameters of the observations where a low frequency QPO has been observed.

The PCA data were obtained in several simultaneous different modes (see Table 3). Only proportional counter units (PCUs) 0 and 2 were always active during our observations. Standard 2 data from these two PCUs were used to create light- and hardness curves for the whole outburst, whereas the high time resolution data from all active PCUs (in a given observation) were used for the timing analysis. A hardness ratio was defined as the ratio of counts in the range $7-15 \mathrm{keV}$ (12-31 channels) to those in the range $2-7 \mathrm{keV}(0-11)$. Fast Fourier Transforms were made from 16s data intervals with Nyquiest frequencies of $64 \mathrm{~Hz}$ (Binned data) and $4096 \mathrm{~Hz}$ (Single bit data). The resulting PDS were averaged, rebinned logarithmically, and the Poissonian noise, including the Very Large Events (VLE) contribution (Zhang 1995; Zhang et al. 1995), was subtracted. The PDS were normalized to fractional squared rms, following Belloni \& Hasinger (1990). PDS fitting was carried out by using the standard Xspec fitting package by using a one-to-one energy-frequency conversion and a unit response. Following Belloni et al. (2002), we fitted the noise components with two Lorentzian shapes, one zerocentered and a second one centered at a few Hz. The QPOs were fitted with one Lorentzian each too, only occasionally needing the addition of a Gaussian component to better approximate the shape of the narrow peaks and to reach values of reduced $\chi^{2}$ close to 1 . For the observations where the dynamical power spectra showed transitions between different power spectral shapes (see below), we separated different time intervals in order to obtain average power spectra for each shape.

For every $16 \mathrm{~s}$ interval we also produced a cross-spectrum between the $2-5$ and $5-13 \mathrm{keV}$ resolved light curves (defined as $C(j)=X_{1}^{*}(j) X_{2}(j)$, where $X_{1}$ and $X_{2}$ are the complex Fourier coefficients for the two energy bands at a frequency $v_{j}$ ), calculated average cross-spectrum vectors for each observation, and then derived a phase lag as a function of frequency from the angle in the complex plane of these vectors $\left(\phi_{j}=\arg [C(j)]\right)$. The error in $\phi$ is computed from the observed variance of $C$ in the real and imaginary directions. In line with recent literature we defined phase lags as positive when the hard X-ray variability follows the soft one. To quantify the phase-lag behaviour of the QPOs, we extracted the phase lags in a range centered at the QPO peak frequency and corresponding to the width of the peak itself $\left(v_{\mathrm{p}} \pm F W H M / 2\right.$, see Reig et al. 2000). In Table 2, we list the frequency, fullwidth half-maximum $(F W H M), 2-15 \mathrm{keV}$ fractional rms, and phase lag of the QPO for each observation in which one was detected. The total integrated fractional rms $(2-15 \mathrm{keV})$ of the PDS is given as well.

In Fig. 1, we show the 2-60 keV light curve of the whole outburst, the hardness ratio and the integrated $0.03-64 \mathrm{~Hz}$ fractional rms of the $2-15 \mathrm{keV}$ light curves.

\section{Power spectra and phase lags}

In many of our observations QPOs were detected, with frequencies ranging from $\sim 1$ to $\sim 9 \mathrm{~Hz}$. Three main types could be distinguished, which, based on their phase lag and coherence properties, could be associated with type-A, -B, and -C QPOs. Example power spectra of each type are shown in Fig. 2. In addition to these three main types we also identified various sub-types, which will be discussed below.

A useful method for differentiating between the three types of PDS is shown in Fig. 3, in which we plot the integrated fractional rms of each PDS versus the centroid frequency of the QPO. Several groups of points can be identified. The first large group of points in the plot, type-C and $\mathrm{C}^{*}$ (see Sects. 3.1 and 3.2), is diagonally spread across the plot, covering the whole frequency range between 1 and $10 \mathrm{~Hz}$ and a large range in rms (7-30\% rms). Another group, type-A (see Sect. 3.3), is clustered around a frequency of $8 \mathrm{~Hz}$ and rms of $2 \%$. Finally a third group is located at a slightly higher rms $(4-6 \%)$ in the 4.5-6.5 Hz range: type-B and "B-Cathedral" (see Sects. 3.4 and 3.5). A more detailed analysis of the correlation between the frequency these three QPO types and the integrated fractional rms in different sources is described in a forthcoming paper (Casella et al. 2004, in prep.). In Fig. 4 we show the light curve, the hardness and the total fractional rms of the first 25 days of the outburst (see Fig. 1 for energy and frequency ranges) and indicate where the three types of QPO appear. In the bottom panel we have marked the three types of QPO and their associated sub-types. In the following paragraphs we describe all these types in detail, characterizing their PDS and phase-lag behaviour.

\subsection{Type-C QPOs}

In the early stages of the outburst (see Table 2 for dates), when the source flux was rising quickly, the PDS showed four main components: a strong (15-30\% rms) flat-topped noise and three harmonically related QPOs (see Fig. 5). The central 1-7 Hz QPO was strong (rms amplitude 6-16\%) and narrow $(Q \sim 7-10)$. Note that in a few cases the addition of a Gaussian component was required, in order to better 
Table 2. Power-spectral classification and variability parameters ${ }^{a}$.

\begin{tabular}{|c|c|c|c|c|c|c|c|c|}
\hline Obs. Id. & Date & MJD & QPO Type & $\begin{array}{l}v_{\mathrm{qpo}} \\
(\mathrm{Hz})\end{array}$ & $\begin{array}{c}F W H M_{\text {qpo }} \\
(\mathrm{Hz})\end{array}$ & $\begin{array}{c}\mathrm{rms}_{\mathrm{qpo}}^{b} \\
\%\end{array}$ & $\begin{array}{l}\text { Phase } \mathrm{Lag}^{c} \\
\quad(\mathrm{rad})\end{array}$ & $\begin{array}{c}0.03-64 \mathrm{~Hz} \mathrm{rms}^{b} \\
(\%)\end{array}$ \\
\hline 40124-01-04-00 & 1999 Oct. 11 & 51462.768 & $\mathrm{C}$ & 1.2 & 0.18 & 16.61 & $0.00 \pm 0.01$ & $29.8 \pm 0.3$ \\
\hline 40124-01-05-00 & 1999 Oct. 12 & 51463.833 & $\mathrm{C}$ & 3.05 & 0.31 & 13.48 & $-0.04 \pm 0.01$ & $24.9 \pm 0.2$ \\
\hline 40124-01-06-00 & 1999 Oct. 13 & 51464.109 & $\mathrm{C}$ & 3.64 & 0.46 & 13.43 & $-0.07 \pm 0.01$ & $22.9 \pm 0.1$ \\
\hline 40124-01-07-00 & “ & 51464.633 & $\mathrm{C}$ & 3.65 & 0.46 & 12.97 & $-0.04 \pm 0.01$ & $23.1 \pm 0.2$ \\
\hline 40124-01-08-00 & 1999 Oct. 14 & 51465.305 & $\mathrm{C}$ & 4.39 & 0.48 & 11.85 & $-0.08 \pm 0.01$ & $20.78 \pm 0.08$ \\
\hline 40124-01-09-00 & “ & 51465.498 & $\mathrm{C}$ & 4.95 & 0.56 & 10.85 & $-0.09 \pm 0.01$ & $19.16 \pm 0.08$ \\
\hline 40124-01-10-00 & “ & 51465.902 & $\mathrm{C}$ & 5.81 & 0.72 & 8.52 & $-0.14 \pm 0.01$ & $16.2 \pm 0.1$ \\
\hline $40124-01-11-00$ & 1999 Oct. 15 & 51466.896 & $\mathrm{C}$ & 5.97 & 0.65 & 8.11 & $-0.18 \pm 0.01$ & $16.0 \pm 0.08$ \\
\hline $40124-01-12-00$ & 1999 Oct. 16 & 51467.581 & $\mathrm{~A}$ & 7.75 & 3.85 & 1.75 & $-0.33 \pm 0.07$ & $2.76 \pm 0.05$ \\
\hline 40124-01-13-00 & “ & 51467.961 & $\mathrm{~B}$ & 6.08 & 0.45 & 2.10 & $0.07 \pm 0.07$ & $4.5 \pm 0.06$ \\
\hline 40124-01-13-00 & “ & 51467.973 & A & 8.42 & 2.885 & 1.62 & $-0.3 \pm 0.1$ & $2.8 \pm 0.1$ \\
\hline 40124-01-14-00 & 1999 Oct. 17 & 51468.427 & $\mathrm{C}^{*}$ & 8.64 & 2.37 & 2.83 & $-0.56 \pm 0.04$ & $7.67 \pm 0.04$ \\
\hline 40124-01-14-00 & “ & 51468.427 & $\mathrm{~B}$ & 6.43 & 0.82 & 3.13 & $0.3 \pm 0.09$ & $6.1 \pm 0.1$ \\
\hline 40122-01-01-03 & 1999 Oct. 18 & 51469.093 & B & 5.96 & 0.75 & 3.04 & $0.23 \pm 0.04$ & $5.02 \pm 0.04$ \\
\hline 40122-01-01-02 & “ & 51469.159 & $\mathrm{~B}$ & 5.96 & 0.85 & 2.52 & $0.13 \pm 0.04$ & $4.83 \pm 0.05$ \\
\hline 40122-01-01-02 & “ & 51469.179 & $\mathrm{~A}$ & 7.85 & 3.26 & 1.79 & $-0.02 \pm 0.1$ & $2.9 \pm 0.1$ \\
\hline 40122-01-01-01 & “ & 51469.226 & A & 7.74 & 5.22 & 1.37 & $-0.84 \pm 0.2$ & $2.1 \pm 0.1$ \\
\hline 40122-01-01-00 & “ & 51469.293 & A & 7.59 & 3.81 & 1.64 & $-0.33 \pm 0.1$ & $2.16 \pm 0.09$ \\
\hline 40122-01-01-00 & “ & 51469.360 & $\mathrm{~B}$ & 5.94 & 0.80 & 2.02 & $0.125 \pm 0.06$ & $4.40 \pm 0.06$ \\
\hline 40124-01-16-00 & “ & 51469.493 & $\mathrm{C}^{*}$ & 8.45 & 3.54 & 3.10 & $-0.49 \pm 0.04$ & $7.69 \pm 0.07$ \\
\hline $40124-01-16-00$ & “ & 51469.517 & $\mathrm{C}^{*}$ & 7.7 & 1.36 & 4.53 & $-0.29 \pm 0.04$ & $8.28 \pm 0.09$ \\
\hline 40124-01-17-00 & “ & 51469.893 & $\mathrm{C}^{*}$ & 7.89 & 1.17 & 3.22 & $-0.41 \pm 0.07$ & $9.6 \pm 0.1$ \\
\hline $40124-01-17-00$ & “ & 51469.899 & $\mathrm{C}^{*}$ & 7.54 & 1.58 & 5.07 & $-0.26 \pm 0.04$ & $10.6 \pm 0.1$ \\
\hline $40124-01-17-00$ & “ & 51469.905 & $\mathrm{C}^{*}$ & 7.27 & 1.19 & 5.46 & $-0.22 \pm 0.04$ & $11.7 \pm 0.1$ \\
\hline 40124-01-18-00 & 1999 Oct. 20 & 51471.024 & $\mathrm{C}$ & 5.88 & 0.42 & 6.98 & $-0.17 \pm 0.015$ & $15.81 \pm 0.07$ \\
\hline $40124-01-19-00$ & " & 51471.224 & $\mathrm{C}$ & 5.18 & 0.61 & 9.10 & $-0.15 \pm 0.015$ & $18.11 \pm 0.07$ \\
\hline 40124-01-20-00 & “ & 51471.890 & $\mathrm{C}$ & 6.47 & 0.80 & 6.59 & $-0.25 \pm 0.025$ & $14.04 \pm 0.07$ \\
\hline $40124-01-21-00$ & 1999 Oct. 21 & 51472.503 & $\mathrm{C}$ & 6.34 & 0.90 & 7.29 & $-0.2 \pm 0.02$ & $14.49 \pm 0.06$ \\
\hline 40124-01-15-00 & 1999 Oct. 22 & 51473.245 & $\mathrm{C}$ & 6.13 & 0.72 & 7.11 & $-0.22 \pm 0.04$ & $15.5 \pm 0.1$ \\
\hline $40124-01-23-00$ & “ & 51473.822 & $\mathrm{C}^{*}$ & 6.92 & 0.93 & 5.68 & $-0.27 \pm 0.03$ & $12.79 \pm 0.09$ \\
\hline 40124-01-23-01 & “ & 51473.890 & $\mathrm{C}^{*}$ & 7.73 & 1.59 & 4.00 & $-0.43 \pm 0.03$ & $9.85 \pm 0.07$ \\
\hline $40124-01-15-02$ & 1999 Oct. 23 & 51474.088 & $\mathrm{C}^{*}$ & 7.39 & 4.52 & 3.63 & $-0.43 \pm 0.03$ & $7.91 \pm 0.08$ \\
\hline $40124-01-24-00$ & “ & 51474.429 & B - Cath. & 5.84 & 0.81 & 3.62 & $0.23 \pm 0.04$ & $6.27 \pm 0.06$ \\
\hline $40124-01-25-00$ & “ & 51474.820 & C & 6.19 & 0.68 & 7.18 & $-0.22 \pm 0.02$ & $14.9 \pm 0.1$ \\
\hline 40124-01-26-00 & 1999 Oct. 24 & 51475.154 & $\mathrm{C}^{*}$ & 7.06 & 0.95 & 5.06 & $-0.29 \pm 0.03$ & $12.28 \pm 0.08$ \\
\hline $40124-01-26-00$ & “ & 51475.166 & $\mathrm{C}^{*}$ & 7.24 & 1.06 & 4.43 & $-0.34 \pm 0.03$ & $11.31 \pm 0.07$ \\
\hline $40124-01-26-00$ & “ & 51475.177 & $\mathrm{C}^{*}$ & 7.73 & 1.36 & 3.54 & $-0.40 \pm 0.03$ & $9.84 \pm 0.07$ \\
\hline $40124-01-26-00$ & “ & 51475.218 & $\mathrm{C}^{*}$ & 7.75 & 1.72 & 3.70 & $-0.45 \pm 0.02$ & $9.29 \pm 0.04$ \\
\hline $40124-01-26-00$ & “ & 51475.252 & $\mathrm{C}^{*}$ & 7.56 & 1.24 & 3.65 & $-0.33 \pm 0.03$ & $10.35 \pm 0.07$ \\
\hline 40124-01-27-00 & “ & 51475.428 & B - Cath. & 5.79 & 0.84 & 4.28 & $0.25 \pm 0.07$ & $6.2 \pm 0.1$ \\
\hline $40124-01-28-00$ & 1999 Oct. 25 & 51476.428 & $\mathrm{C}^{*}$ & 7.64 & 2.1 & 2.91 & $-0.48 \pm 0.03$ & $8.69 \pm 0.06$ \\
\hline 40124-01-28-01 & “ & 51476.501 & $\mathrm{C}^{*}$ & 7.52 & 1.53 & 4.22 & $-0.36 \pm 0.04$ & $10.27 \pm 0.08$ \\
\hline 40124-01-29-00 & 1999 Oct. 26 & 51477.152 & $\mathrm{C}^{*}$ & 7.54 & 2.4 & 3.66 & $-0.42 \pm 0.02$ & $8.99 \pm 0.04$ \\
\hline 40124-01-30-00 & 1999 Oct. 27 & 51478.017 & $\mathrm{~B}$ & 5.06 & 0.7 & 3.79 & $0.17 \pm 0.04$ & $5.36 \pm 0.05$ \\
\hline 40124-01-31-00 & “ & 51478.777 & $\mathrm{C}^{*}$ & 7.26 & 0.96 & 3.97 & $-0.33 \pm 0.03$ & $11.12 \pm 0.07$ \\
\hline $40122-01-02-00$ & “ & 51478.816 & $\mathrm{C}^{*}$ & 7.77 & 1.31 & 2.74 & $-0.40 \pm 0.05$ & $9.51 \pm 0.08$ \\
\hline 40122-01-02-00 & “ & 51478.844 & $\mathrm{C}^{*}$ & 7.77 & 1.89 & 3.38 & $-0.44 \pm 0.05$ & $8.9 \pm 0.1$ \\
\hline $40122-01-02-00$ & “ & 51478.882 & $\mathrm{C}^{*}$ & 7.38 & 1.25 & 4.00 & $-0.36 \pm 0.04$ & $10.72 \pm 0.09$ \\
\hline $40122-01-02-00$ & “ & 51478.913 & $\mathrm{C}^{*}$ & 7.54 & 1.39 & 3.59 & $-0.40 \pm 0.04$ & $10.45 \pm 0.08$ \\
\hline $40122-01-02-00$ & “ & 51478.949 & $\mathrm{C}^{*}$ & 7.04 & 0.96 & 4.84 & $-0.37 \pm 0.03$ & $11.99 \pm 0.07$ \\
\hline $40122-01-02-00$ & “ & 51478.980 & $\mathrm{C}^{*}$ & 7.46 & 1.03 & 3.12 & $-0.49 \pm 0.05$ & $10.01 \pm 0.08$ \\
\hline $40124-01-36-00$ & 1999 Nov. 01 & 51483.117 & $\mathrm{~B}$ & 4.69 & 0.63 & 4.06 & $0.16 \pm 0.03$ & $5.58 \pm 0.07$ \\
\hline 40124-01-36-00 & “ & 51483.158 & $\mathrm{~B}$ & 4.69 & 0.54 & 3.93 & $0.22 \pm 0.04$ & $5.88 \pm 0.06$ \\
\hline 40124-01-37-00 & “ & 51483.945 & $\mathrm{~B}$ & 4.59 & 0.54 & 4.31 & $0.16 \pm 0.03$ & $5.80 \pm 0.05$ \\
\hline $40124-01-37-01$ & 1999 Nov. 02 & 51484.077 & B & 4.53 & 0.62 & 4.05 & $0.07 \pm 0.05$ & $4.89 \pm 0.15$ \\
\hline 40124-01-37-02 & “ & 51484.276 & $\mathrm{~B}$ & 4.45 & 0.61 & 4.03 & $0.09 \pm 0.03$ & $4.33 \pm 0.06$ \\
\hline
\end{tabular}


Table 2. Continued.

\begin{tabular}{ccccccccc}
\hline \hline Obs. Id. & Date & MJD & QPO Type & $\begin{array}{c}v_{\text {qpo }} \\
(\mathrm{Hz})\end{array}$ & $\begin{array}{c}F W H M_{\text {qpo }} \\
(\mathrm{Hz})\end{array}$ & $\begin{array}{c}\mathrm{rms}_{\mathrm{qpo}}^{b} \\
\%\end{array}$ & $\begin{array}{c}\text { Phase Lag }^{c} \\
(\mathrm{rad})\end{array}$ & $\begin{array}{c}0.03-64 \mathrm{~Hz} \mathrm{rms}^{b} \\
(\%)\end{array}$ \\
\hline 40124-01-39-00 & “ & 51485.875 & $?$ & 5.61 & 2.3 & 2.68 & $-0.51 \pm 0.04$ & $4.1 \pm 0.1$ \\
40124-01-40-00 & 1999 Nov. 04 & 51486.828 & $?$ & 5.37 & 1.7 & 3.00 & $-0.43 \pm 0.07$ & $3.1 \pm 0.3$ \\
$40124-01-40-01$ & “ & 51486.873 & $?$ & 5.22 & 1.3 & 2.89 & $-0.3 \pm 0.1$ & $4.0 \pm 0.2$ \\
40124-01-41-00 & 1999 Nov. 05 & 51487.009 & $?$ & 4.74 & 1.3 & 2.18 & $-0.42 \pm 0.05$ & $3.5 \pm 0.1$ \\
40124-01-42-00 & 1999 Nov. 06 & 51488.409 & $?$ & 7.08 & 2.4 & 1.75 & $-0.88 \pm 0.06$ & $4.46 \pm 0.08$ \\
40124-01-49-01 & 1999 Nov. 15 & 51497.255 & $?$ & 5.21 & 1.9 & 2.82 & $-0.36 \pm 0.07$ & $4.1 \pm 0.2$ \\
40124-01-58-01 & 1999 Dec. 15 & 51527.004 & $?$ & 9.1 & 0.8 & 2.17 & $0.0 \pm 0.2$ & $8.9 \pm 0.2$ \\
40124-01-59-00 & 1999 Dec. 18 & 51530.133 & $?$ & 8.15 & 2.1 & 2.91 & $0.0 \pm 0.2$ & $6.71 \pm 0.25$ \\
40124-01-61-00 & 2000 Jan. 03 & 51546.036 & $?$ & 4.65 & 0.8 & 2.3 & $-0.17 \pm 0.25$ & $4.3 \pm 0.4$ \\
\hline
\end{tabular}

${ }^{a}$ Only observations with evidence for low frequency QPO are listed.

${ }^{b}$ Normalization according to Belloni \& Hasinger (1990).

${ }^{c}$ Phase lag in radians between the $2-5$ and $5-13 \mathrm{keV}$ light curves and integrated over the frequency range $v_{\mathrm{qpo}} \pm F W H M / 2$.

Table 3. RXTE/PCA data modes active during the XTE J1859+226 observations.

\begin{tabular}{llcc}
\hline \hline $\begin{array}{l}\text { Mode } \\
\text { name }\end{array}$ & $\begin{array}{l}\text { Time res. } \\
(\mathrm{s})\end{array}$ & $\begin{array}{c}\text { Number of } \\
\text { PHA channels }\end{array}$ & $\begin{array}{c}\text { PHA } \\
\text { Energy range }(\mathrm{keV})\end{array}$ \\
\hline Standard1 & $2^{-3}$ & 1 & $2-60$ \\
Standard2 & $2^{4}$ & 129 & $2-60$ \\
Binned & $2^{-7}$ & 36 & $2-15$ \\
SB1 & $2^{-13}$ & 14 & $2-6$ \\
SB2 & $2^{-13}$ & 22 & $6-15$ \\
Event & $2^{-16}$ & 16 & $15-60$ \\
\hline
\end{tabular}

approximate the peak's shape. This was also the case for the second harmonic, which was always present. A broad $(Q \sim 2)$ subharmonic was also detected in all cases, except for the first observation (40124-01-04-00) where the frequency of the fundamental was at its lowest value). The harmonic relation between the three peaks was confirmed by allowing the centroids to vary independently. We decided to fix the centroid frequencies to harmonic ratios to obtain self-consistent estimates of the amplitude and width.

The phase-lag behaviour of the type-C power spectra is similar to that found in GRS $1915+105$ by Reig et al. (2000). It is strongly correlated with the frequency of the QPO, with a trend towards negative lags for increasing QPO frequency. Figure 6 (all panels except the bottom one) shows four examples covering the whole observed range in QPO frequency. Owing to poor statistics, lags became unmeasurable at high frequencies; we thus plot them only below $20 \mathrm{~Hz}$. In all observed cases, the fundamental of the QPO showed negative lags (see Fig. 17 and the discussion), with a clear trend towards zero for decreasing centroid frequency, consistent with the Reig et al. (2000) results on GRS 1915+105 (where peak lags are positive at frequencies below $\sim 1 \mathrm{~Hz}$ ). The lags of subharmonic peak were always negative as well, while the second harmonic always showed positive lags. It is important to mention the absence in the cross spectra of narrow features standing out at the frequency of the QPO and harmonics peaks. This suggests that

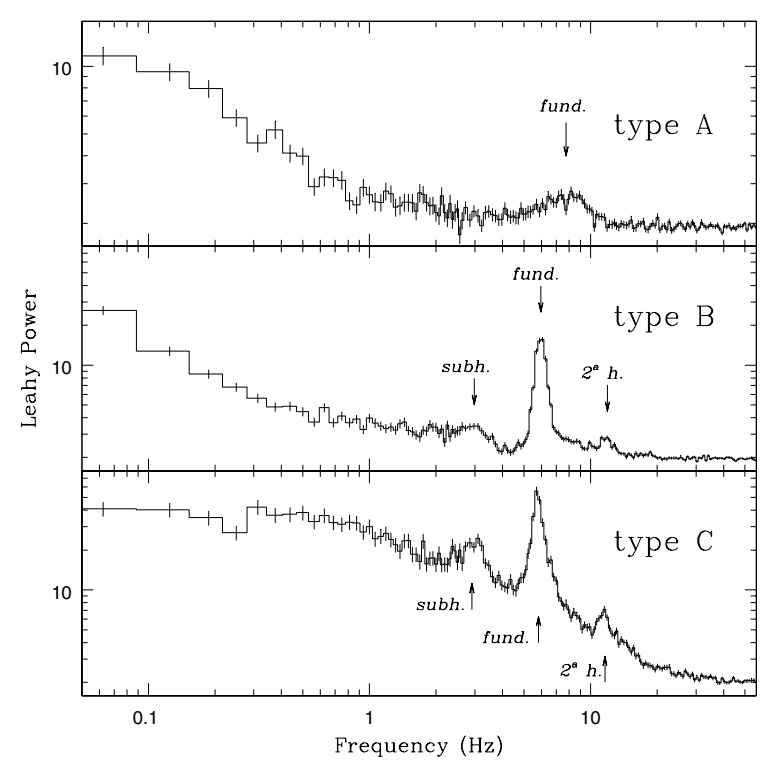

Fig. 2. Examples of type A, B and C QPOs from our XTE J1859+226 observations. QPO and harmonics peaks are indicated. Upper panel: Obs. 40124-01-12-00. Middle panel: Obs. 40122-01-01-03. Bottom panel: Obs. 40124-01-10-00). The Poisson noise was not subtracted.

we are measuring the phase-lags of the underlying noise continuum rather than the phase lags associated with the QPO peaks.

\subsection{Type-C* QPOs}

Later in the outburst, type-C QPOs appeared again (see Table 2). In this case, however, the centroid frequency was higher and the rms amplitude lower. We refer to these QPOs as a type- $\mathrm{C}^{*}$. The power spectra showed a strong red-noise component and a broad QPO peak at a centroid frequency of 7-9 Hz (Fig. 7, upper panel). Again a Gaussian component was added in many cases in order to better approximate the peak shape. A second harmonic peak was sometimes present, as well a subharmonic one. Phase lags were negative and large, up to $10 \mathrm{~ms}$, over the range $v_{\mathrm{p}} \pm F W H M / 2$, and decreased 


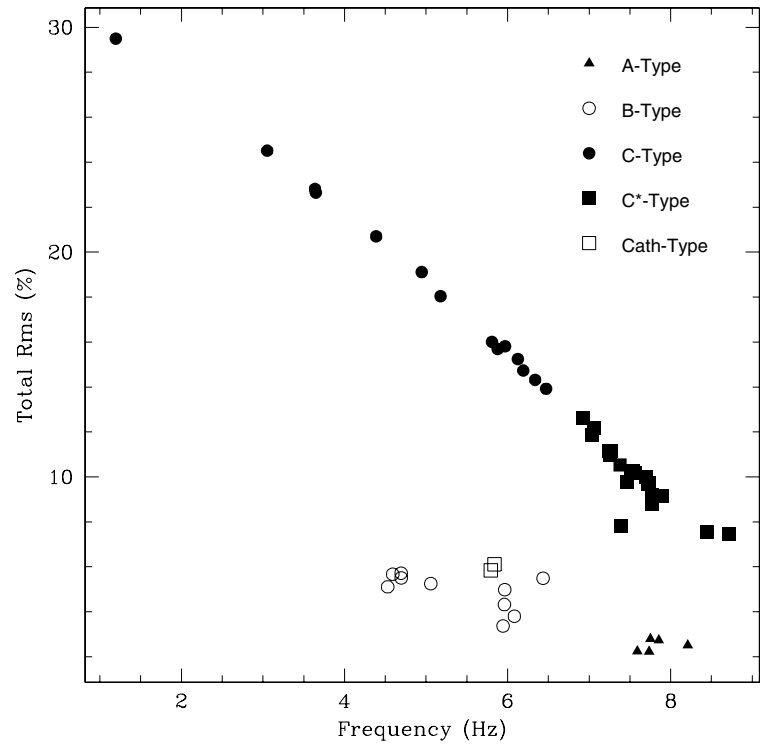

Fig. 3. Centroid QPO frequency vs. $0.03-64 \mathrm{~Hz}$ fractional $\mathrm{rms}$ of the detected QPOs. Each point corresponds to a different observation, except for the few cases in which transitions between different pds shapes have been observed. Error bars are smaller than symbols.

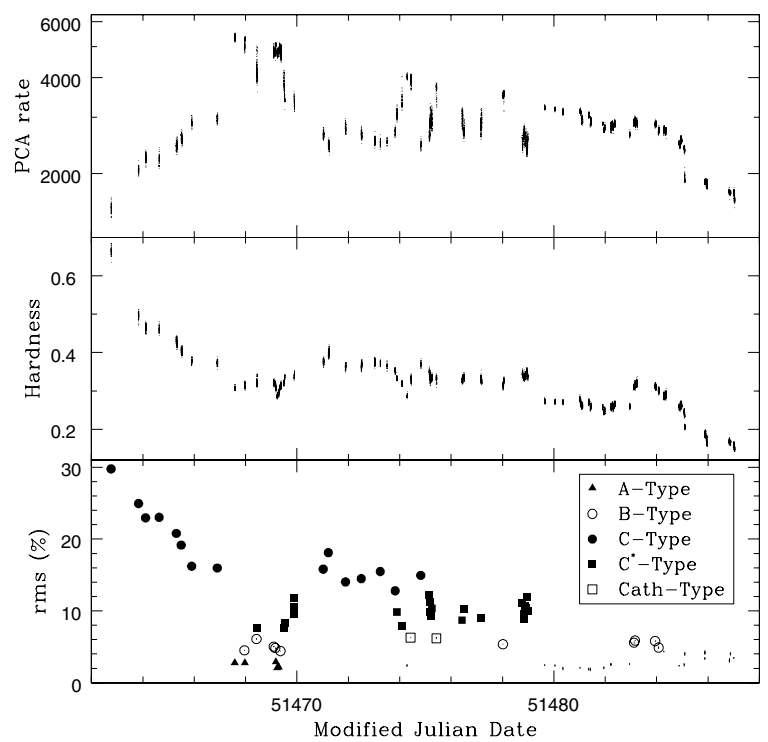

Fig. 4. Same as Fig. 1, but for only the first 25 days of the outburst. See inset for the symbols that are used to represent different QPO types in the lower panel - dots are used if no QPO was detected.

rapidly at frequencies slightly higher than the QPO centroid, as can be seen in Fig. 7 (bottom panel).

Even though the QPO centroid frequency range was different from that of type-C QPOs, and the rms and $Q$-values were smaller, the results above (see also Fig. 3) provide evidence that the properties of type-C and type- $\mathrm{C}^{*} \mathrm{QPOs}$ are smoothly connected if ordered for increasing QPO frequency. The phase-lag behaviour confirms this: even though the shape of a "normal" type-C QPO (Fig. 6, upper panel) was rather different from that of type $C^{*}$ QPOs (Fig. 6, bottom panel), a continuous transition between the two took place for increasing QPO frequency.

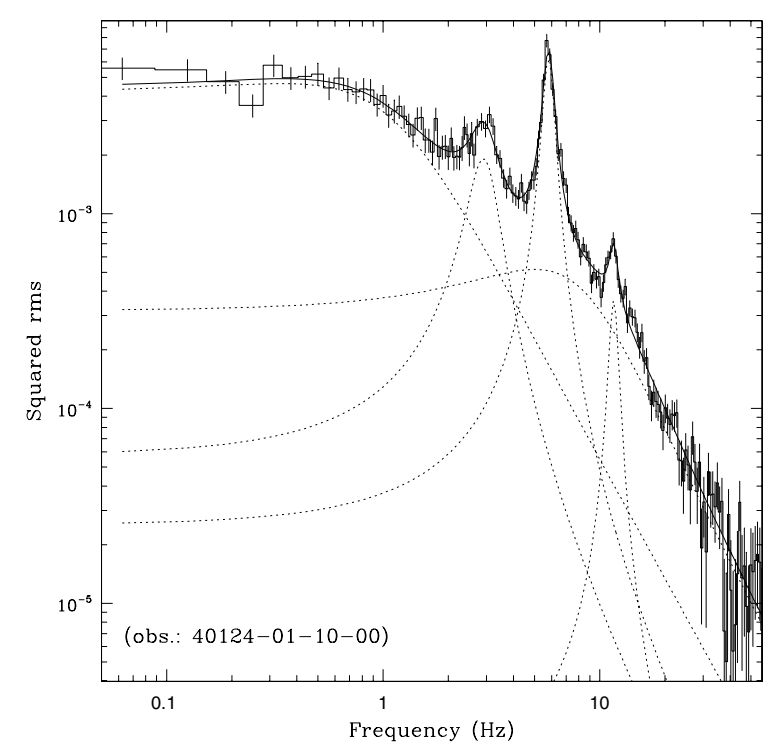

Fig. 5. An example of type-C power spectrum (2-15 keV; Obs.: 40124-01-10-00). The solid line shows the best fit with five Lorentzians (dotted lines). See the correspondent lags in Fig. 6, fourth panel.

However, owing to the long time scale of the QPO frequency variability (not detectable during one single RXTE observation) it was not possible to observe a direct transition between the two QPO types.

\subsection{Type-A QPOs}

Type-A power spectra (see an example in Fig. 8, upper panel) appeared at the peak of the outburst (see Table 2), when the count rate was very high. They were characterized by a broad QPO $(Q \sim 2)$, with centroid frequencies between 7.5 and $8.5 \mathrm{~Hz}$ and fractional rms around $\sim 1.5 \%$, and a low amplitude (few \% rms) red-noise component. Neither a subharmonic nor a second harmonic was present. This was the QPO type with the lowest total rms in our sample. The phase lags, except for a negative excess around the QPO frequency, were consistent with zero.

\subsection{Type-B QPOs}

The narrow $(Q \sim 10)$ QPO characterizing this type of PDS appeared only in a rather restricted frequency range between 4.5 and $6.5 \mathrm{~Hz}$ (see an example in Fig. 9, upper panel). The rednoise was very weak (few \% rms). The QPO peak profile was often more similar to a Gaussian than a Lorentzian, but we needed to combine both components in order to obtain a good fit (it is worth noticing that a similar combination was used by Homan et al. (2001) and Wijnands et al. (1999) for the XTE J1550-564 data, and by Nespoli et al. (2003) for the GX 339-4 data. In the latter case the authors explained the Gaussian shape with the presence of centroid frequency variability on a $\sim 10 \mathrm{~s}$ time scale. However, such variability was not detected in the XTE J1859+226 data). A weak second harmonic was always present, while a subharmonic 


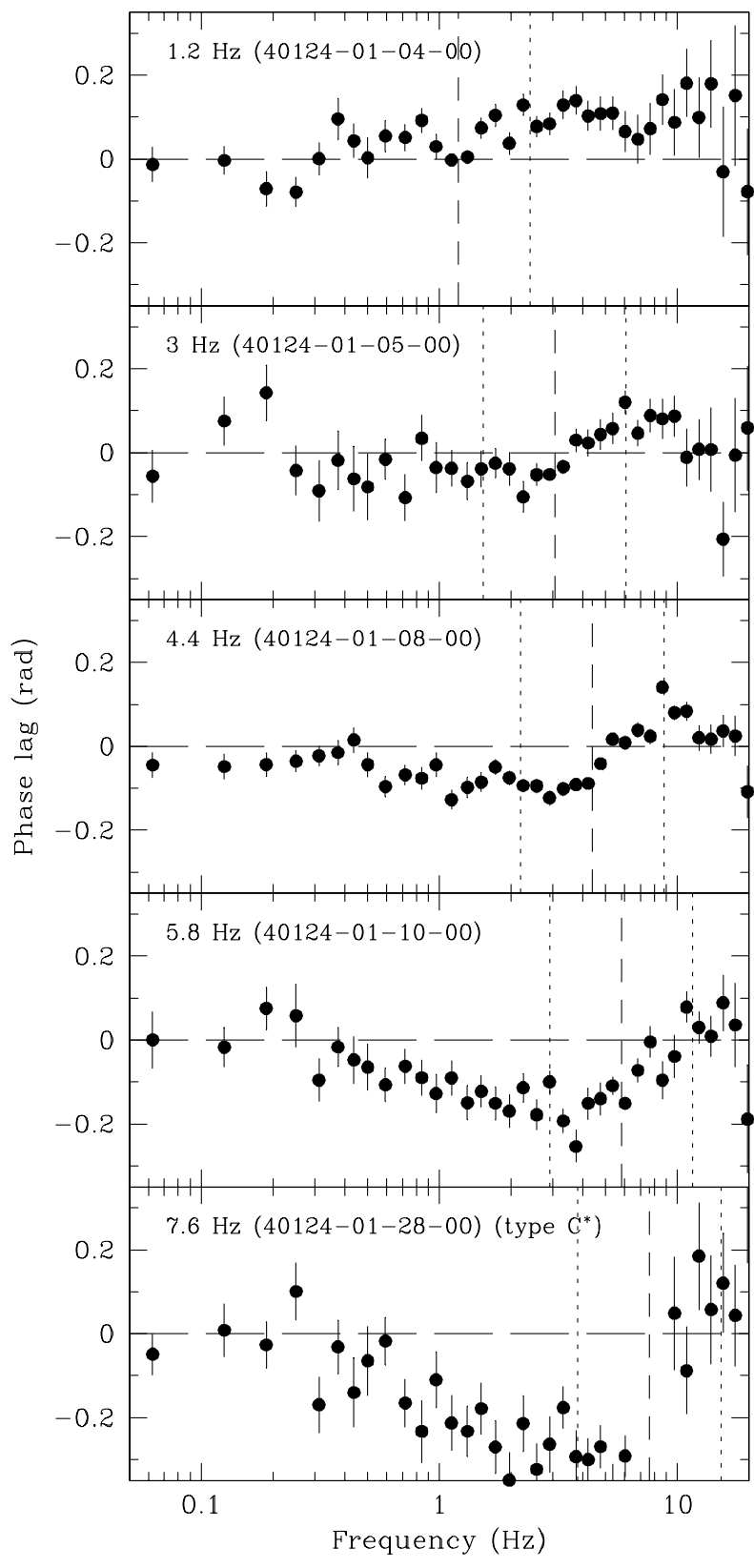

Fig. 6. Type-C phase lags vs. frequency for four different QPO frequencies. Bottom panel: an example of type- $\mathrm{C}^{*}$ phase lags. Positive values indicate that the hard $(5-13 \mathrm{keV})$ photons are lagging the soft $(2-5 \mathrm{keV})$ photons. QPO centroid frequency and observation I.D. are indicated for each panel. The dashed lines mark the frequency of the QPO, while the dotted lines mark the subharmonic (if present) and second harmonic frequencies.

appeared (with rms amplitudes $<1 \%$ ) only when the fundamental frequency was at its highest values and the fundamental and the second harmonic had low amplitudes. This behaviour suggests the presence of a sort of "balance" between the amplitude of the three peaks, particularly between the second harmonic and subharmonic: when the subharmonic peak was at its highest rms the second harmonic is not present, and vice versa. Unfortunately, poor statistics did not allow a more precise assessment of this. The lags (see Fig. 9, bottom panel) were positive at the frequency of the fundamental peak (see Fig. 18

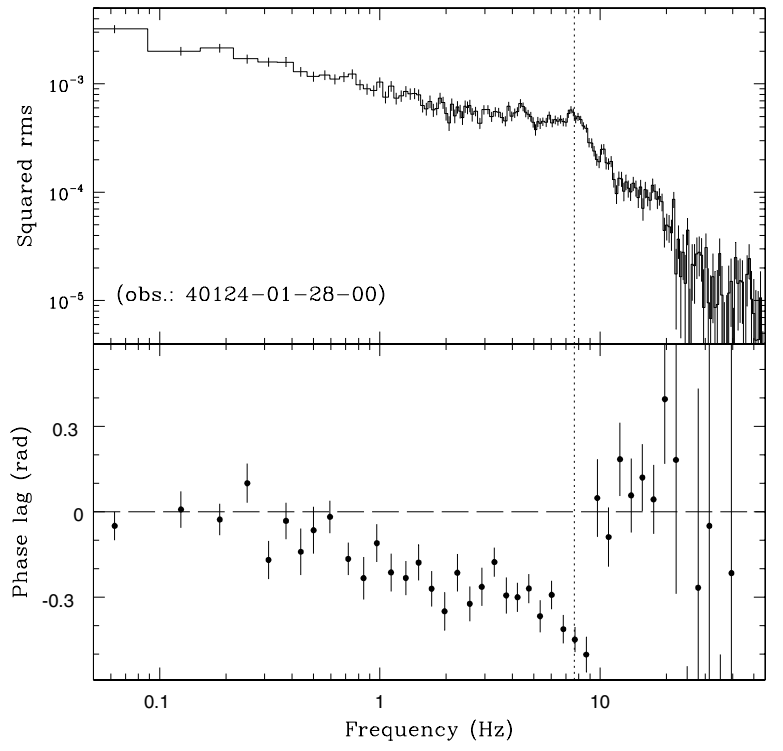

Fig. 7. An example of type $C^{*}$ PDS $(2-15 \mathrm{keV})$ and phase lags (Obs.: 40124-01-28-00). The dotted vertical line marks the frequency of the QPO.

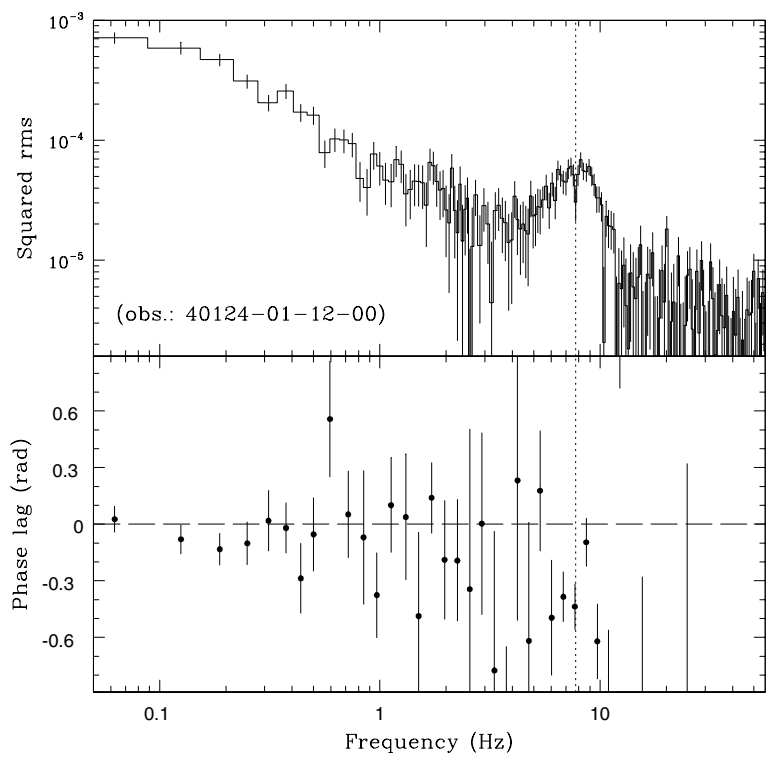

Fig. 8. An example of type-A PDS (2-15 keV) and phase lags (Obs.: 40124-01-12-00). The dotted vertical line marks the frequency of the QPO. Lags at frequencies above $\sim 10 \mathrm{~Hz}$ are, given the lack of statistics, random scattering between $-\pi$ and $\pi$.

for values) and negative at the frequencies of subharmonic and second harmonic.

\subsection{Type B-“Cathedral" QPOs}

During two observations, 40124-01-24-00 (MJD: 51 474.429) and 40124-01-27-00 (MJD: 51 475.428), the power spectrum showed a peculiar double-peaked QPO (see Fig. 10, upper panel), similar to some of the type-B QPOs observed in XTE J1550-564 (Wijnands et al. 1999; Homan et al. 2001). In both observations, two strong and narrow peaks were observed at harmonically related frequencies of $\sim 3$ and $6 \mathrm{~Hz}$. 


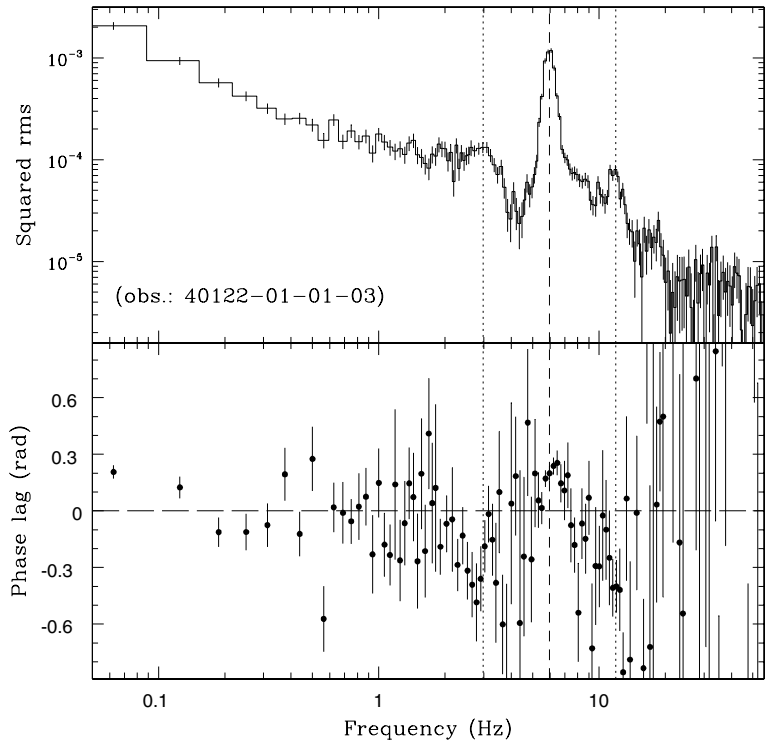

Fig. 9. An example of type-B PDS $(2-15 \mathrm{keV})$ and phase lags (Obs.: 40122-01-01-03). The dashed vertical line marks the frequency of the QPO, while the dotted lines mark the subharmonic and second harmonic frequencies.

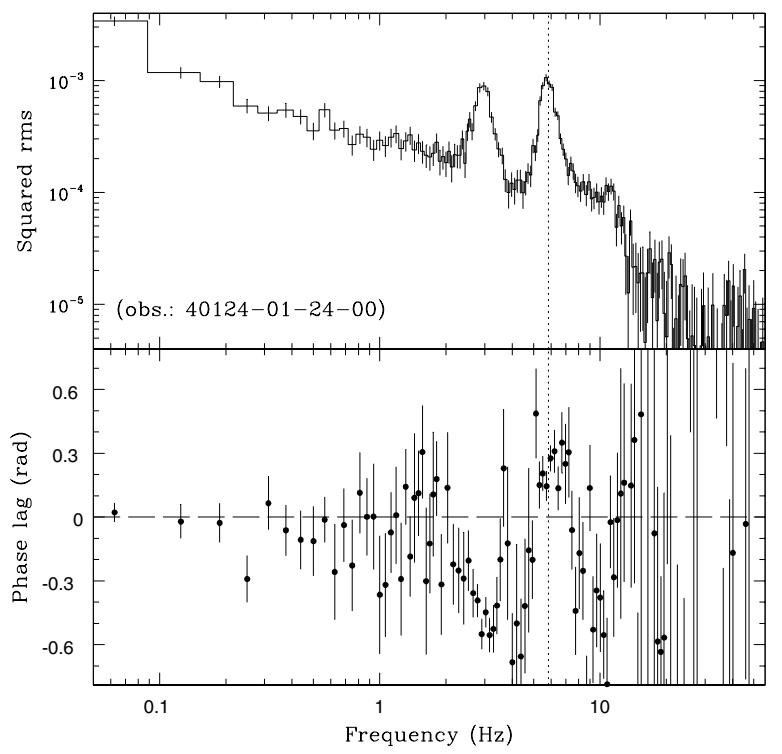

Fig. 10. An example of type B-"Cathedral" PDS (2-15 keV) and phase lags (Obs.: 40124-01-24-00). The dotted vertical line marks the frequency of the QPO.

In the case of the second observation we needed to add a Gaussian component to both peaks in order to better approximate their shape. A weak red-noise component was present (the two Lorentzians having $\sim 2 \% \mathrm{rms}$ each). The rms amplitudes of the two QPO peaks were $\sim 4 \%$ and $2-2.5 \%$, for the $6 \mathrm{~Hz}$ and $3 \mathrm{~Hz}$ QPO, respectively. A weak ( $\sim 1 \% \mathrm{rms})$ peak at the harmonically related frequency of $12 \mathrm{~Hz}$ was observed in both observations. The phase lags were consistent with zero over the whole frequency range except at the frequencies where the QPOs were seen $(3$ and $6 \mathrm{~Hz}$ ). The lags of the $3 \mathrm{~Hz}$ QPO were negative and corresponded (calculated, according with our definition, in the range $v_{\mathrm{p}} \pm F W H M / 2$ ) to a delay

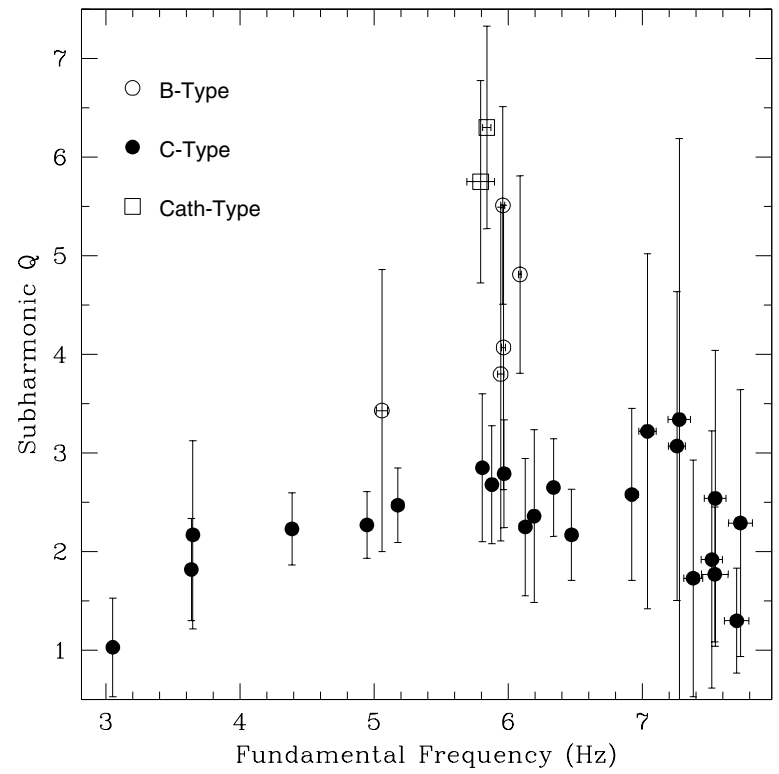

Fig. 11. Coherence of the subharmonic peak for different pds types.

of $\sim 20 \mathrm{~ms}$ in the soft X-ray variations, while for the $\sim 6 \mathrm{~Hz}$ peak the lags were positive and corresponded to a delay of $\sim 7 \mathrm{~ms}$ in the hard X-ray variations.

One might classify the $\sim 3 \mathrm{~Hz}$ peak as the fundamental and the $\sim 6 \mathrm{~Hz}$ peak as the second harmonic. In this case, the $\sim 12 \mathrm{~Hz}$ would be the fourth harmonic, while the third harmonic would be missing or too weak to be detected. However, a comparison of this "cathedral" power spectrum with the others, suggests a similarity with the type-B power spectrum. Apart from the high amplitude and narrower width of the $\sim 3 \mathrm{~Hz}$ peak, the general characteristics are almost identical: the total rms was around a few $\%$ (compared to $\sim 15 \%$ for the type-C spectrum when the QPO was at $\sim 6 \mathrm{~Hz}$ ), and both the red-noise and the $\sim 6 \mathrm{~Hz}$ peak were weak. Moreover, type-B PDS showed positive lags for the fundamental, as opposed to those in the type-C power spectra. Thus it seems natural to identify the $\sim 6 \mathrm{~Hz}$ peak (having positive lags) as the fundamental, the $\sim 12 \mathrm{~Hz}$ as its second harmonic, and the $\sim 3 \mathrm{~Hz}$ peak as its subharmonic. This is also in line with other PDS types in which we identified subharmonic peaks. Moreover, the integrated rms of the subharmonic peak of the B-cathedral PDS $(\sim 2-3 \%)$ was not much higher than that of other PDS. On the contrary, it was lower than that of type-C QPOs (between 3 and $8 \%$ ), and only slightly higher than that of "normal" type-B QPOs ( 1-2\%). However the coherence of the B-cathedral subharmonic peak was the largest observed among all low frequency QPOs (see Fig. 11), making the feature more prominent. It is worth noticing that the $\sim 6 \mathrm{~Hz}$ QPO frequency clearly stands out in Fig. 11 (see the discussion).

\subsection{Special cases: Unclassified QPOs}

In a few of the observations following the last type-B PDS (MJD 51484 ) we detected QPOs that we were not able to classify in terms of the type $\mathrm{A} / \mathrm{B} / \mathrm{C}$ scheme. Owing to poor statistics and a too high centroid frequency variability, we could not 


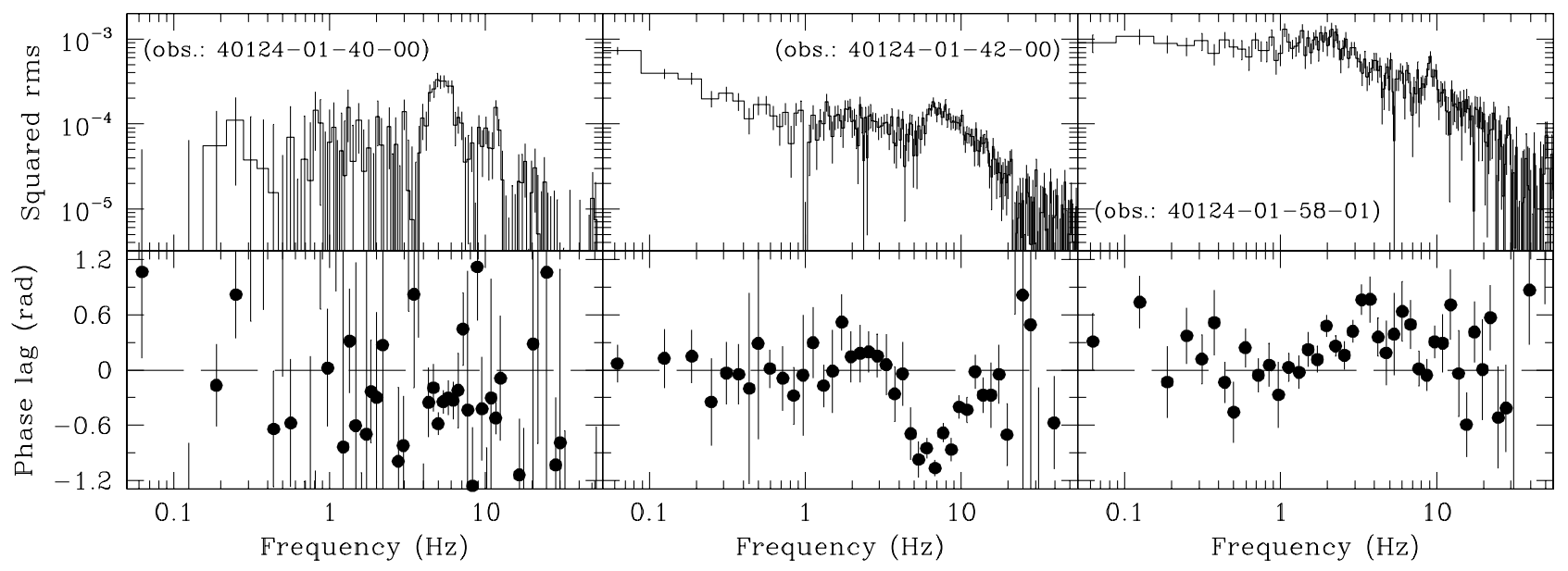

Fig. 12. Three examples of unclassified power spectra $(2-15 \mathrm{keV})$ with their phase lags.

unambiguously fit the power spectra and then obtain characteristic parameters. However, for sake of completeness we report in Table 2 the best estimate for the parameters of each observation. In Fig. 12 we show three representative examples of power spectra and phase lags from these observations.

\section{Timing and spectral evolution}

In some observations, the dynamical PDS showed rapid (a few tens of seconds) transitions between different power spectral shapes. In all cases, the transitions involve type-B QPOs. In panels a-c of Fig. 13 we show three examples of different behaviours. In the first half of the observation 40124-01-13-00 (MJD 51467.961, panel a), when the light curve was highly variable, the PDS was of type-B (with a QPO frequency $\sim 6 \mathrm{~Hz}$ ). Simultaneously with the rise observed in the light curve after $\sim 1100 \mathrm{~s}$ from the start, the PDS showed a sharp transition to a type-A shape with a QPO frequency of $\sim 8 \mathrm{~Hz}$ (not visible in the gray scale representation). In the second part of the observation, the light curve was much less variable and had a higher mean count rate. Notice that a brief interval with the same characteristics (type-A QPO, higher flux) was seen $\sim 200$ s into the light curve, again with very sharp in and out transitions.

For observation 40124-01-14-00 (MJD 51468.427, panel b) the behaviour was different: when the source flux was low the power spectrum showed a type-C shape $(\sim 8.7 \mathrm{~Hz})$, while during the two peaks in count-rate, when it reached values close to those of the first half of the previous observation, the PDS was of type-B $(\sim 6.4 \mathrm{~Hz})$. The transitions were again very sharp. In observation 40122-01-01-00 (MJD 51 469.360, panel c) the source showed the opposite behaviour: at high count rates, the power spectrum transitioned to type-A $(\sim 7.6 \mathrm{~Hz})$, while at lower fluxes a type-B PDS was observed ( $\sim 6 \mathrm{~Hz}$ ), similar to panel (a). This was clearly seen before and after the gap in the middle of the observation.

It is worth remarking that in all cases the transitions involve type-B QPOs. When the source showed a type-B PDS and underwent a fast transition to a lower count rate, the PDS changed to type-C; when on the other hand the transition was to a higher count rate, the PDS changed to typeA. In the same way, fast transitions from type-A to type-B and from type-C to type-B always involved a decrease and increase in count rate, respectively. Direct transitions between types $\mathrm{A}$ and $\mathrm{C}$ were not observed. Panel (d) of Fig. 13 (Obs. Id. 40124-01-24-00, MJD 51474.429), shows a fourth type of rapid transition, which occurred when the source showed a B-Cathedral type PDS. Corresponding to the dips in the light curve, the PDS changed its shape, with the two peaks partially losing their coherence while the red noise increased. Unfortunately, the time intervals in which this happened were too short for a detailed power-spectrum analysis.

In Fig. 14 we plot a light curve of all observations in which fast transitions were observed. From this figure it is evident that the count rate at which the transitions occur follow an exponential trend. Type-B QPOs appear in a narrow count rate range, as can be seen in the inset of the same figure, where we show the entire portion of the outburst where low frequency QPOs were detected.

Similar fast transitions between different types of QPO and broad-band noise components were reported with Ginga from GS 1124-68 (Takizawa et al. 1997) and GX 339-4 itself (Miyamoto et al. 1991). Interestingly, also in these cases the sharp QPO has a frequency around $\sim 6 \mathrm{~Hz}$.

Finally, in Fig. 15 (Obs. Id. 40124-01-38-01), we show a peculiar event: the source count rate dropped by $\sim 20 \%$ (with an integrated fractional $\mathrm{rms}$ of $\sim 2.5 \%$ before and $\sim 4.0 \%$ after the drop) in about $100 \mathrm{~s}$. The increase in rms occurs mainly above $5 \mathrm{~Hz}$, with a broad power excess clearly visible around $\sim 6-7 \mathrm{~Hz}$ (not shown). The hardness distribution was also different, as can be seen in the bottom panel of Fig. 15. During the observation, the hard and soft colors decreased fairly continuosly, but simultaneously with the drop in count rate the soft color showed a clear discontinuity. This sharp transition is also clearly visible in Fig. 1 (MJD: 51 485.075) where it can be seen that after the drop the count rate kept following an approximate exponential trend for several days, with a slope close to that shown in Fig. 4 before the drop, which was thus a conspicuous feature in the overall light curve of the outburst. 

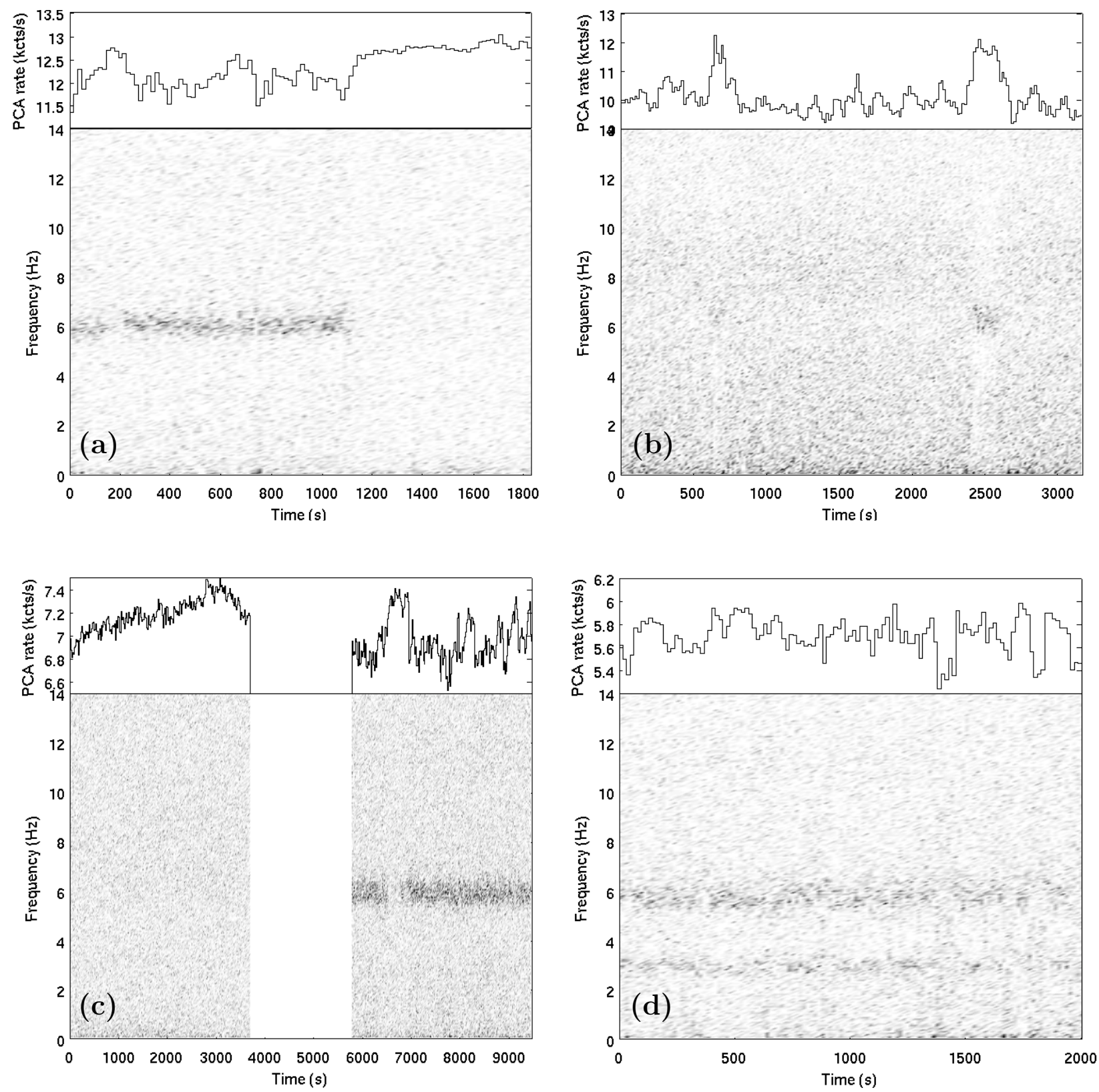

Fig. 13. Light curves and dynamical PDS for observations 40124-01-13-00 (panel a), all five PCUs on), 40124-01-14-00 (panel b), five PCUs on), 40122-01-01-00 (panel c), three PCUs on) and 40124-01-24-00 (panel d), three PCUs on). The lower power value of the typeA QPO peaks with respect to the type-B render them invisible in the dynamical power spectra of panels a) and c). However, these are clearly seen in the total average PDS.

\section{Discussion}

We analyzed the RXTE/PCA data from the 1999 outburst of the black hole candidate XTE J1859+226, studying the low frequency QPOs and their detailed behaviour. We could classify most of QPOs in three main types (A, B, C), plus a couple of sub-types, obtaining a coherent scenario which can be compared to that of other systems such as XTE J1550-564, for which the "ABC" classification was introduced (Wijnands et al. 1999; Remillard et al. 2002b), and GX 339-4, where a type-B QPO was found (Nespoli et al. 2003). We now discuss our results in terms of relations between derived quantities.
- Energy dependence: in Fig. 16, we show the fractional rms of the QPO as a function of energy for type-A, B and $\mathrm{C}$ spectra. The rms amplitude of the QPOs increases with energy and then flattens above $\sim 10 \mathrm{keV}$. The similarity of the trend in the three QPO types, particularly in the $\mathrm{B}$ and $\mathrm{C}$ cases, is apparent. The energy spectra of black hole candidates are often described in terms of two components, one soft/low energy component and another hard/high energy component (see e.g. Tanaka \& Lewin 1995). The observed energy dependence of the fractional rms clearly leads to the conclusion that the QPOs are associated with the high-energy component. Notice that a scenario in which the intrinsic fractional rms of the QPOs 


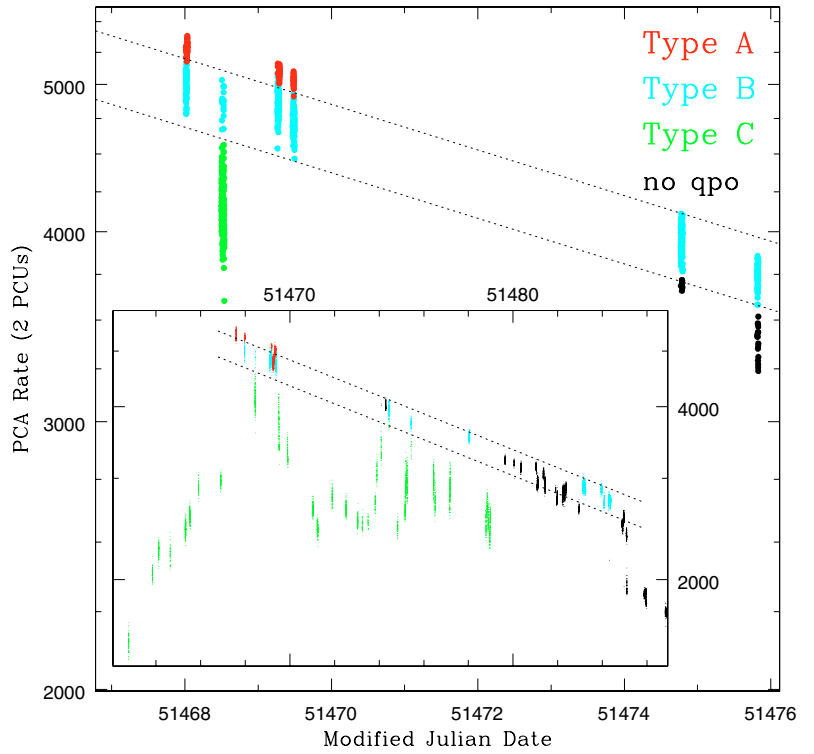

Fig. 14. Light curve of the observations showing transitions between two different QPO types. The two parallel lines have been drawn so as to intersect the transition points. In the inset the lines have been extended to the part of the outburst where QPOs appear. Different grayscales indicate different QPO types. (A color image is available on line) Black points correspond to data in which no QPOs were found.

(i.e. the rms normalized only to the source counts from the high energy component) is constant - with the observed decrease in the rms towards lower energy being caused by a non-variable soft component - cannot be ruled out. If this hypothesis were true, the QPOs could then be interpreted as bare flux oscillations of the high energy component only, without photon index changes. Nevertheless, in GRS 1915+105 a similar energy dependence for the type-C QPO was observed, but the analysis of higherenergy HEXTE data showed that the QPO rms decreases above $20 \mathrm{keV}$ (Tomsick \& Kaaret 2001). Rodriguez et al. (2004) found however that this cut-off was not always present, and proposed an explanation in terms of a contribution from the jets to the hard X-ray component. If this contribution is significant, and the QPOs are unrelated to the jet, its presence would thus produce the observed cutoff in the energy dependence of the QPO rms.

- Time lags: in Fig. 17, we show the behaviour of the time lags of the fundamental QPO peak as a function of the total rms of the PDS. A separation among the three different QPO types is evident: type-C QPOs show a strong and well defined trend, with smaller soft lags for larger rms, while type-B QPOs have hard lags and are clustered around rms of a few percent. The latter show also evidence for a dependence of the time-lags on the rms. Finally, type-A lags are negative and show large scatter. The separation of the three types is evident also in Fig. 18 (left panel), where we plot the time lags of the fundamental and of the second harmonic. Type-B and type-C QPOs show a similar behaviour: the lags of the fundamental and those of the second harmonic, with the exception of a few cases with large error bars, always have opposite signs, resulting in a clustering
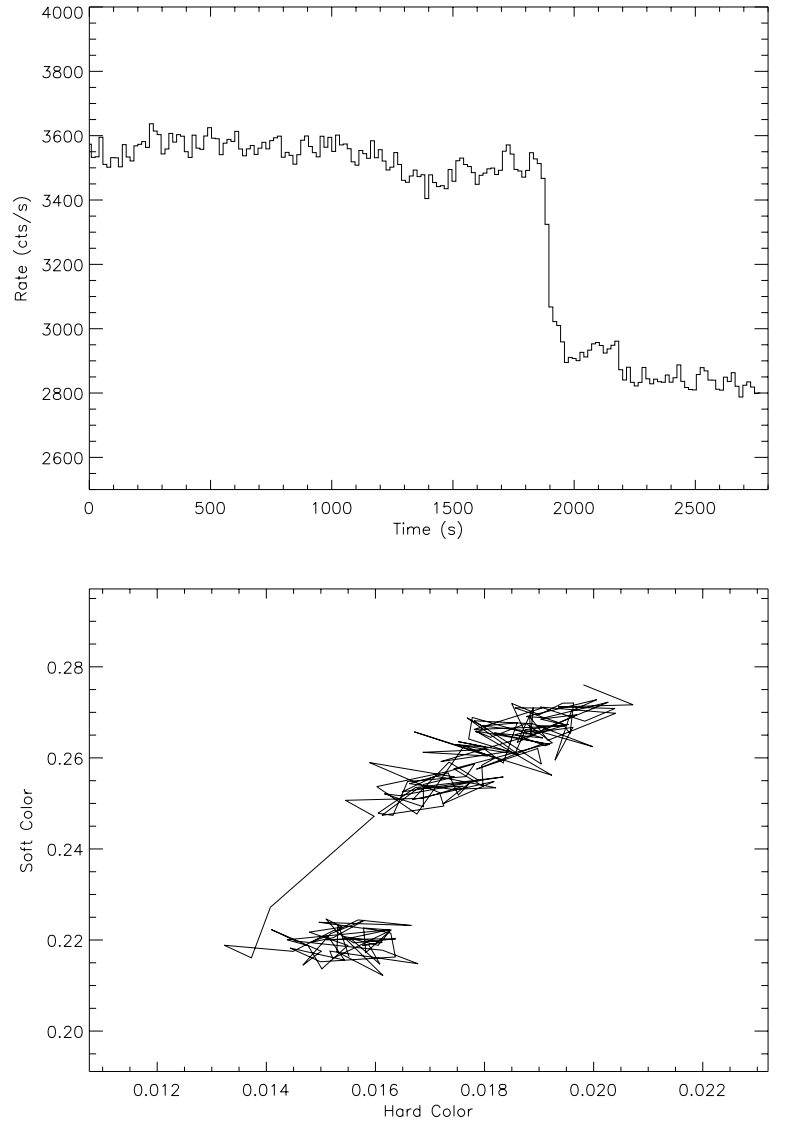

Fig. 15. Light curve and color-color diagram for observation 4012401-38-01. Only three PCUs were on (MJD: 51 485.075).

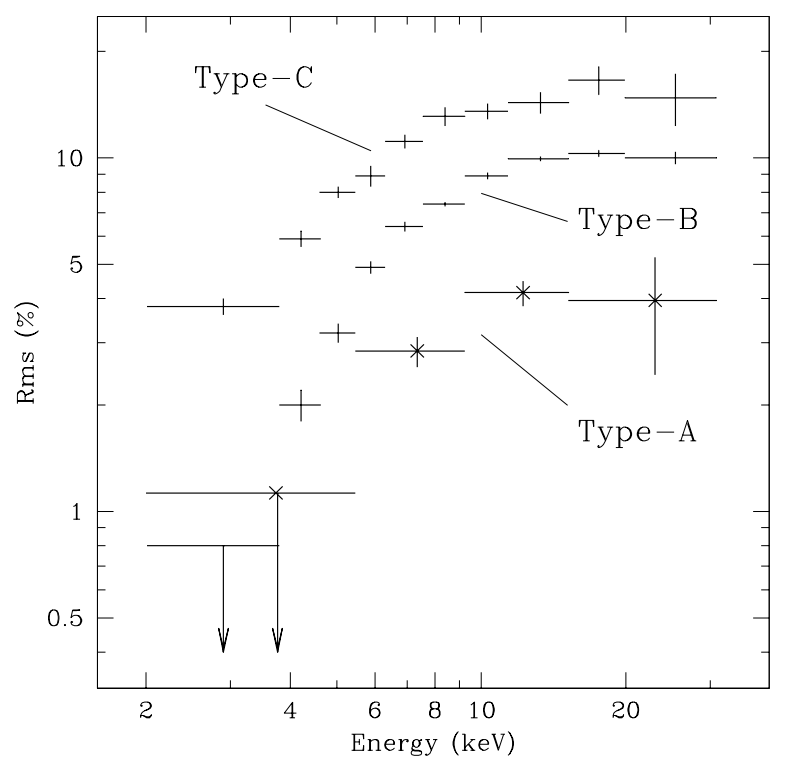

Fig. 16. Fractional rms amplitude of the fundamental QPO peak as a function of energy for QPO types A (stars, obs: 40124-01-12-00), B (40122-01-01-03) and C (40124-01-21-00).

of the points in the second and fourth quadrant. This analysis could not be extended to type-A QPOs, since they don't show any harmonic peak.

This peculiar pattern in the time lags of the different harmonic components was already noticed by Remillard et al. (2002b) in the PDS of XTE J1550-564. Different lags in 


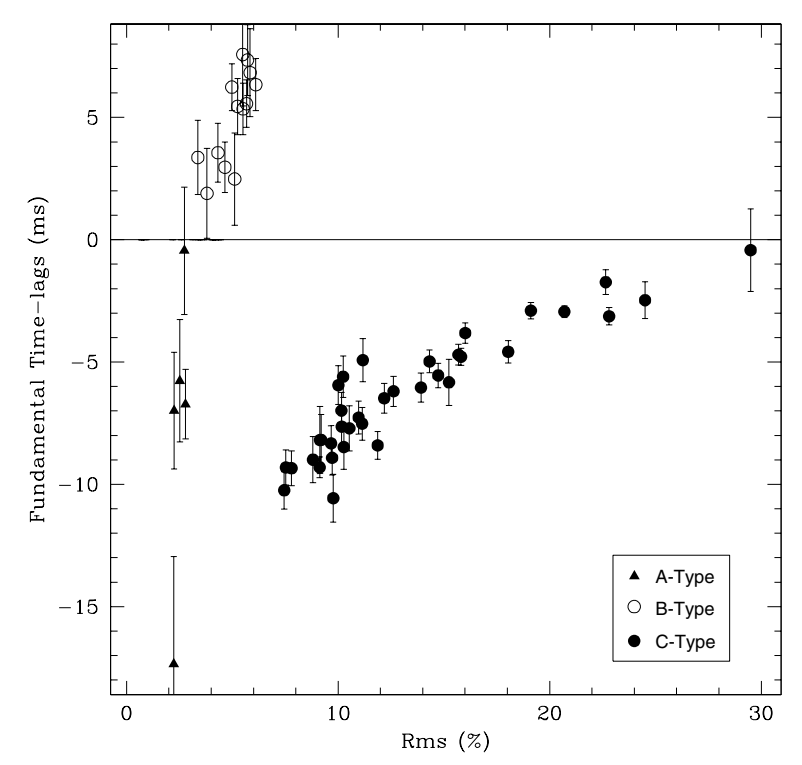

Fig. 17. Total fractional rms $(0.03-64 \mathrm{~Hz})$ vs. time-lags of the fundamental QPO peak.

these components are difficult to interpret. It is of course possible that the oscillations at different harmonics have a different physical origin, while connected to the same underlying "clock", in which case the difference in sign of time lags would be naturally associated to these different physical mechanisms. However, if one assumes the more natural scenario in which the harmonic content of the QPO is simply the result of the non-sinusoidal shape of the oscillation, the higher harmonics do no have a physical meaning by themselves (for a discussion of the subharmonic see below). Such strong differences in time lags would then indicate that the shape of the oscillation is different at different energies, in a way which is highly reproducible. In order to understand this phenomenon in detail, a theoretical model for the shape of the oscillation is needed.

- Subharmonic peaks: the right panel of Fig. 18, giving the time lags of the subharmonic versus those of the fundamental, shows that subharmonic lags are always negative. This behaviour is independent of the QPO type and thus of the sign of time lags at the fundamental and second harmonic peak frequencies. This somehow makes the subharmonic oscillations stand out, and suggests a common origin for it in both B and C QPO types.

The existence of a subharmonic peak opens then a serious issue about the physical mechanism that would produce them. Even though there are other astrophysical examples of subharmonics (see e.g. Aikawa \& Antonello 2000 for the case of Cepheids and Masser \& Tagger 1997 for the case of disk galaxies), there are still no generally-accepted physical explanations. A basic mathematical explanation involves a subharmonic resonance, which can arise in forced nonlinear oscillators. It can be shown (see for example Butikov 2002) that for such an oscillator a subharmonic resonance of order $n$ can occur when the driving frequency is close to an integer multiple $n$ of the natural fundamental frequency. In this scenario, the frequency of $\sim 6 \mathrm{~Hz}$ seems to play an important role: from Fig. 11 it is clear that the coherence of the type-B subharmonic peak is higher when the fundamental peak is close to $6 \mathrm{~Hz}$. It is worth noticing however that type-C QPOs do not show any peculiar behavior when at this frequency. In a forthcoming paper we shall discuss in greater detail the role of this frequency in different classes of sources, BHCs (see e.g. Nespoli et al. 2003), Z-sources and atoll-sources (see e.g. Belloni et al. 2004).

There is of course an alternative scenario in which the frequency of the subharmonic peak is in reality the fundamental frequency of the system. This hypothesis would find some support in the fact that for all types of QPOs the subharmonic always shows the same sign of lags. If this is the case, there would be no need for an explanation of subharmonic peaks; however, the subharmonic is not always observed and almost never (with the exception of the "cathedral" cases) the strongest peak. Moreover, any model under this scenario would have to explain the absence of the third harmonic.

- Color evolution: in Fig. 19 we plot the Hardness-Intensity diagram of the outburst, which shows clear analogies with diagrams of other BHCs (see Homan et al. 2001; Belloni 2003; Rossi et al. 2003). The energy spectrum is hard at the beginning of the outburst, quickly softening as the count rate approaches the outburst peak. Here, the flux shows strong variations, hardening and softening on short time scales. It is during this phase, at the highest count rates, that the QPOs were nearly always present. In particular, from the right panel of Fig. 19 it can be seen that type-A QPOs appear when the hardness indicates that the energy spectrum is softer than when type-C QPOs are present, while type-B QPOs appear at hardness values overlapping those of type-C and type-A QPOs, but always restricted between 0.2-0.3. A similar correlation between QPO types and hardness was found in XTE J1550-564 (Homan et al. 2001). From the result reported in Sect. 4, it is thus clear that the type-B QPO can be associated with spectral transitions. This result is also found in GX 339-4 (Belloni et al. 2004 , in prep.). Either this oscillation is excited in a small range of disk parameters (accretion rate?), or its frequency depends only weakly on these parameters. In either cases, this oscillation seems to be an important ingredient in the evolution of the outbursts of a number of BHT.

After this phase, the count rate first decreased while the spectrum still softens, then it showed a hard flaring episode, after which it continued to decrease while maintaining roughly a constant hardness. Finally, after more than four months from the beginning of the outburst, the energy spectrum hardened, moving towards hardness values comparable to those of the first observation. Following Homan \& Belloni (in prep.), the roughly horizontal branches at the top and at the bottom of Fig. 19 would therefore correspond to the very high/intermediate state (see Homan et al. 2001), appearing at different flux values, while the vertical branch to the left would represent the high/soft state (as can be inferred also from the low rms values). Since the early part of the rise of the outburst has not been observed we can only argue, in analogy with other sources, that the source moved 

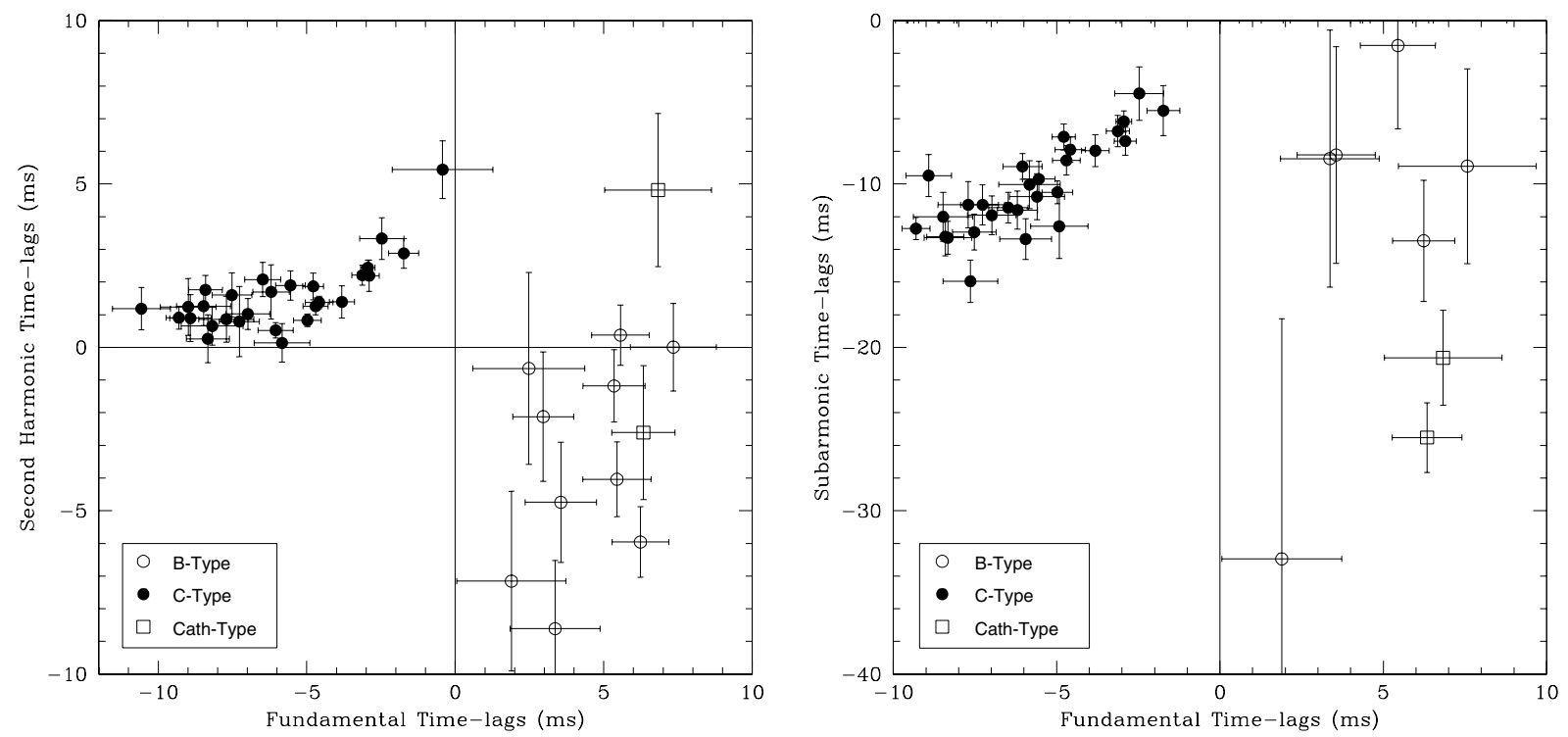

Fig. 18. Time-lags of the fundamental QPO peak vs. time-lags of its second harmonic (left panel) and subharmonic (right panel).

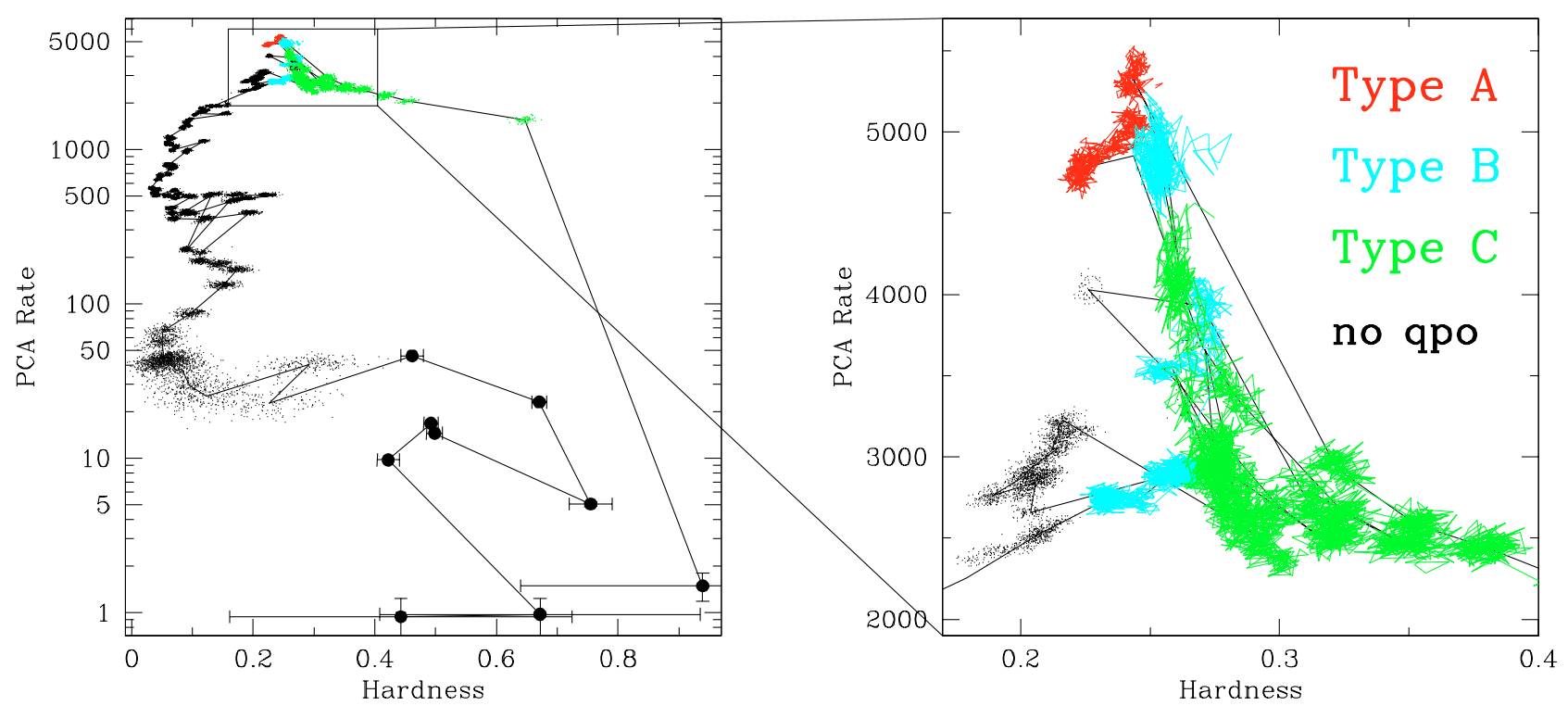

Fig. 19. Left panel: Hardness-Intensity diagram (see Fig. 1 for variables definition). The time resolution is $16 \mathrm{~s}$ except for the first and the last eight observations, which have one point for each observation in order to improve the statistics. Different grayscales indicate different QPO types. (A color image is available on line) Right panel: enlargement of the region of the diagram where most of type A-B-C QPOs appear.

along a roughly vertical branch on the right, which would correspond to the hard state.

\section{Conclusions}

From the large set of observations presented here, the classification of low-frequency QPOs into three types emerges strengthened and is extended to another source, XTE J1859+226. In particular, the type B and C QPOs seem to be a key ingredient that is found in a number of sources. Their different properties (while at the same centroid frequency of $\sim 6 \mathrm{~Hz}$ ) might provide clues to the understanding of the physical mechanisms during the VHS/IS of BHCs and are probably related to the two "flavors" of VHS/IS observed in these systems (see e.g. Miyamoto et al. 1993). The observed energy dependence, that rules out a direct disk origin, is an important constrain for the future physical identification of these features. The difference in time-lag behaviour of the different peaks and the presence of the subharmonic peak are challenging features of the QPO phenomenon. In particular our results provide additional evidence that the frequency of $\sim 6 \mathrm{~Hz}$ is related to some fundamental process. The evidence of a threshold triggering the $\sim 6 \mathrm{~Hz}$ QPO, superimposed to the roughly exponential decay of the outburst, furthermore suggests the existence of a second parameter in addition to the mass accretion rate probably responsible for the long time-scale evolution of the source.

In addition, after the analysis of yet another transient source, it is clear that the outbursts of these systems, in their 
general evolution, show strong similarities. This should be examined in the light of theoretical models for the high-energy emission and possibly even used as another identification tool for these sources.

Acknowledgements. This work was partially supported by MIUR under CO-FIN grant 2002027145. Jeroen Homan acknowledges support from NASA.

\section{References}

Aikawa, T., \& Antonello, E. 2000, A\&A, 363, 593

Belloni, T., \& Hasinger, G. 1990, A\&A, 230, 103

Belloni, T., van der Klis, M., Lewin, W. H. G., et al. 1997, A\&A, 322, 857

Belloni, T., Psaltis, D., \& van der Klis, M. 2002, ApJ, 572, 392

Belloni, T. 2003, in Proc. of the II BeppoSAX Meeting: The Restless High-Energy Universe (Amsterdam, May 5-8, 2003), ed. E. P. J. van den Heuvel, J. J. M. in 't Zand, \& R. A. M. J. Wijers, to appear

Belloni, T., Parolin, I., \& Casella, P. 2004, A\&A, 423, 969

Brocksopp, C., Fender, R. P., McCollough, M., et al. 2002, MNRAS, 331,765

Butikov, E. I. 2002, J. Phys. A: Math. Gen., 35, 6209

Cui, W. 1998, in High Energy Processes in Accreting Black Holes, ed. J. Poutanen, \& R. Svensson (Graftavallen, Sweden), June 1998 [arXiv: astro-ph/9809408]

Cui, W., Zhang, S. N., Chen, W., \& Morgan, E. H. 1999, ApJ, 512, 43

Cui, W., Shrader, C. R., Haswell, C. A., \& Hynes, R. I. 2000, ApJ, 535, L123

Dieters, S. W., Belloni, T., Kuulkers, E., et al. 2000, ApJ, 538, 307

Focke, W. B., Markwardt, C. B., Swank, J. H., \& Taam, R. E. 2000, in Rossi 2000: Astrophysics with the Rossi X-ray Timing Explorer, Greenbelt, March 2000, E104

Garnavich, P. M., Stanek, K. Z., \& Berlind, P. 1999, IAU Circ., 7276

Garnavich, P. M., \& Quinn, J. 2000, IAU Circ., 7388

Homan, J., Wijnands, R., van der Klis, M., et al. 2001, ApJS, 132, 377

Markwardt, C. B., Marshall, F. E., \& Swank, J. H. 1999, IAUC, 7274

Markwardt, C. B., Focke, W. B., \& Swank, J. H. 2001, in X-ray Emission from Accretion onto Black Holes, ed. T. Yaqoob, \& J. H. Krolik, Johns Hopkins University, Baltimore, published electronically

Masser, F., \& Tagger, M. 1997, A\&A, 322, 442

McClintock, J. E., \& Remillard, R. A. 2004, in Compact Stellar X-ray Sources, ed. W. H. G. Lewin, \& M. van der Klis (Cambridge: Cambridge University Press), to appear

Mendez, M., \& van der Klis, M. 1997, ApJ, 479, 926
Mendez, M., Belloni, T., \& van der Klis, M. 1998, ApJ, 499, L187

Miyamoto, S., Kimura, K., Kitamoto, S., Dotani, T., \& Ebisawa, K. 1991, ApJ, 383, 784

Miyamoto, S., Iga, S., Kitamoto, S., \& Kamado, Y. 1993, ApJ, 403, L39

Nespoli, E., Belloni, T., Homan, J., et al. 2003, A\&A, 412, 235

Park, S. Q., Miller, J. M., McClintock, J. E., et al. 2003, ApJ, submitted

Pooley, G. G., \& Hjellming, R. M. 1999, IAU Circ., 7278

Reig, P., Belloni, T., van der Klis, M., et al. 2000, ApJ, 541, 883

Remillard, R. A., Morgan, E. H., McClintock, J. E., Bailyn, C. D., \& Orosz, J. A. 1999, ApJ, 522, 397

Remillard, R. A., Muno, M. P., McClintock, J. E., \& Orosz, J. A. 2002a, in New Views on Microquasars, 49

Remillard, R. A., Sobczak, G. J., Muno, M. P., \& McClintock, J. E. 2002b, ApJ, 564, 962

Rodriguez, J., Fuchs, Y., Hannaikainen, D., et al. 2004, Proc. of the 5h INTEGRAL workshop, (Munich, Feb. 16-20, 2004), to be published by EDP

Rossi, S., Homan, J., Miller, J. M., \& Belloni, T. 2003, in Proc. of the II BeppoSAX Meeting: The Restless High-Energy Universe (Amsterdam, May 5-8, 2003), ed. E. P. J. van den Heuvel, J. J. M. in 't Zand, \& R. A. M. J. Wijers, to appear

Sobczak, G. J., McClintock, J. E., Remillard, R. A., et al. 2000, ApJ, 531,537

Takizawa, M., Dotani, T., Mitsuda, K., et al. 1997, ApJ, 489, 272

Tanaka, Y., \& Lewin, W. H. G. 1995, in X-ray binaries, ed. W. H. G. Lewin, J. van Paradijs, \& E. P. J. van den Heuvel (Cambridge: Cambridge University Press), 126

Tomsick, J. A., \& Kaaret, P. 2000, ApJ, 537, 448

Tomsick, J. A., \& Kaaret, P. 2001, ApJ, 548, 401

van der Klis, M. 1995, in X-ray binaries, ed. W. H. G. Lewin, J. van Paradijs, \& E. P. J. van den Heuvel (Cambridge: Cambridge University Press), 252

van der Klis, M. 2000, ARA\&A, 38, 717

van der Klis, M. 2004, in Compact Stellar X-ray Sources, ed. W. H. G. Lewin, \& M. van der Klis (Cambridge: Cambridge University Press), to appear

Wijnands, R., Homan, J., \& van der Klis, M. 1999, ApJ, 526, L33

Wijnands, R., Mendez, M., Miller, J. M., \& Homan, J. 2001, MNRAS, 328,451

Wood, A., Smith, D. A., Marshall, F. E., \& Swank, J. 1999, IAUC, 7274

Zhang, W. 1995, XTE/PCA Internal Memo

Zhang, W., Jahoda, K., Swank, J. H., Morgan, E. H., \& Giles, A. B. 1995, ApJ, 449, 930

Zurita, C., Sánchez-Fernández, C., Casares, J., et al. 2002, MNRAS, 334, 999 